\title{
The Euler-Riemann Gases, and Partition Identities
}

\author{
NouredDine Chair \\ Physics Department, The University of Jordan, Amman, Jordan \\ Email: n.chair@ju.edu.jo
}

\begin{abstract}
The Euler theorem in partition theory and its generalization are derived from a non-interacting quantum field theory in which each bosonic mode with a given frequency is equivalent to a sum of bosonic mode whose frequency is twice ( $s$-times) as much, and a fermionic (parafermionic) mode with the same frequency. Explicit formulas for the graded parafermionic partition functions are obtained, and the inverse of the graded partition function (IGPPF), turns out to be bosonic (fermionic) partition function depending on the parity of the order $s$ of the parafermions. It is also shown that these partition functions are generating functions of partitions of integers with restrictions, the Euler generating function is identified with the inverse of the graded parafermionic partition function of order 2. As a result we obtain new sequences of partitions of integers with given restrictions. If the parity of the order $s$ is even, then mixing a system of parafermions with a system whose partition function is (IGPPF), results in a system of fermions and bosons. On the other hand, if the parity of $s$ is odd, then, the system we obtain is still a mixture of fermions and bosons but the corresponding Fock space of states is truncated. It turns out that these partition functions are given in terms of the Jacobi theta function $\theta_{4}$, and generate sequences in partition theory. Our partition functions coincide with the overpartitions of Corteel and Lovejoy, and jagged partitions in conformal field theory. Also, The partition functions obtained are related to the Ramond characters of the superconformal minimal models, and in the counting of the MooreRead edge spectra that appear in the fractional quantum Hall effect. The different partition functions for the Riemann gas that are the counter parts of the Euler gas are obtained by a simple change of variables. In particular the counter part of the Jacobi Theta function is $\frac{\zeta(2 t)}{\zeta(t)^{2}}$. Finally, We propose two formulas which brings the additive number theory and the multiplicative number theory closer.
\end{abstract}




\section{INTRODUCTION}

In arithmetic quantum theories the spectrum is chosen to be logarithmic in order to connect quantum mechanics to number theory (multiplicative number theory), since the partition function is related to the Riemann zeta function or other Dirichlet series [1] and to string theory [3]. In these theories, number theoretic identities have been derived and interpreted. Spector in 44 obtained the expressions for the fermionic and parafermionic partition function in terms of a bosonic partition function using the notion of partial supersymmetry. Although his expressions for the partition functions are written correctly, his assumption that the powers of bosonic operators are bosonic operators turns out to be false. This problem is fixed by saying that the spectrum of a single bosonic mode (harmonic oscillator) is a sum of a spectrum of an even harmonic oscillator and that of a fermionic oscillator. Keeping this interpretation in mind, a non-interacting quantum theory free of a logarithmic spectrum is then considered and shown to be connected to the Euler generating function for partitions. As a consequence, the quantum theory we considered is connected with the additive number theory [5]. In this paper, the Riemann gas is also considered by relating the different partition functions of Euler gas additively computed with those of the Riemann gas that has logarithmic spectrum. We have derived the Euler theorem by expressing the fermionic partition function in terms of the bosonic partition function, the Euler theorem says that the number of partitions of a number $k$ (a positive integer) containing odd numbers only equals the number of partitions of a number $k$ without duplication. It turns out that the square of a bosonic operator is related to a parafermion whose order is not an integer [6]. The commutation relations satisfied by these operators is a modification of the Green's trilinear relations [7]. Therefore, the quantum theory that we should consider is constructed in such a way that it does not contain the square of the bosonic operators. The bosonic partition function of a single bosonic oscillator in this case, is the sum of two parafermionic partition functions, one with parastatistics order $s=1 / 2$ and the other with order $s=3 / 2$. This sum then tuns into the product of a bosonic and fermionic partition functions at different temperatures. Similarly, from parafermionic partition function we have derived another identity in partition theory [8] which equates the number of partitions of $k$ in which no part appears more than $s-1$ times, with the number of partitions of $k$ such that no part is divisible by $s$. Using this identity we prove Andrews' result [8] in connection with generating functions that exclude squares and their generalizations [9]. By considering the graded parafermionic case, some explicit formulas for the partition functions are obtained. It turns out that the partition functions that have positive norms are the inverse of the graded parafermionic partition functions (IGPPF). These partition functions correspond to the generalized Euler generating function for partitions and give rise to sequences of partitions of integers that have appeared in [10]. The Euler's generating function of partitions has the interpretation of being the inverse of the graded fermionic partition function. This is the partition function of the Euler gas, it will be shown that its generalization is a bosonic (fermionic) partition function depending on the parity of the order $s$ of parafermions. Mixing a parafermionic system with a system whose partition function is (IGPPF) results in mixing fermions with bosons. The corresponding Fock space of the states associated to this mixture is a tensor product (convolution) of the fermoinic and the bosonic Fock spaces. This happens when the order of parafermions is even, however, if the order is odd, then the mixing is still made of fermions and bosons but the corresponding Fock space of states is truncated. It turns out that these partition functions are related to the Jacobi theta function and have a meaning in partition theory and are identical to the theory of overpartitions [11], or jagged partitions in conformal field theory [12] and their restrictions. These partition functions are related to the Ramond characters of the superconformal minimal models [13, and in the counting of the Moore-Read edge spectra that appear in the fractional quantum 
Hall effect [14]. The different partition functions obtained in this paper are related to generating functions of partitions of integers. therefore, we may call these theories additive quantum theories to differentiate them from multiplicative quantum theories whose partition functions are related to the Riemann zeta function. By using the fact that bosonic partition functions for the Riemann gas and the Euler gas are given by products. Then, it is possible to go from the additive quantum theory to multiplicative quantum theory, and write down all the different partition functions for the Riemann gas by a simple change of variables. All these partition functions are written in terms of the Riemann zeta functions, or alternatively, written as Dirichlet series. Our paper is organized as follows, in sections 2 and 3, the Euler theorem in partition theory and its generalization are proved by using a non-interacting quantum field theory. Here, in this theory, each bosonic mode of a given frequency is considered as a sum of a bosonic mode whose frequency is twice ( $s$-times) as much and fermionic (parafermionic) mode with the same frequency. In Section 4, the graded parafermionc partition function, i.e, generalization of the graded fermionic partition function, and the inverse of the graded parafermionic partition functions are considered. These partition functions are bosonic (fermionic) partition functions depending on the parity of the order $s$ of the parafermion. Here, we gave a number of examples of generating function of sequences of partitions, some of which are the outcome of the present work. In Section 5, partition functions of a mixed system made of parafermions system and bosonic (fermionic ) system are shown to be related the Jacobi Theta function, $\theta_{4}(0, x)$. The partition functions of the Riemann gas and their relation to those of the Euler gas are discussed in great details in Section 6, in particular, it is shown that the counter

part of the partition function $\frac{1}{\theta_{4}(0, x)}$ in the additive quantum theory, is $\frac{\zeta(t)^{2}}{\zeta(2 t)}$. $\theta_{4}$ is like the theta function $\theta$ is in the ordinary variables additive in nature, while the Dirichlet and in particular the Riemann zeta function are multiplicative. The Mellin transform is used to translate additive into multiplicative and vice versa. It is well known that the Riemann zeta function is the Mellin transform of $(\theta(0, x)-1)$. A simple exercise shows that the alternating Riemann zeta function or the Dirichlet eta function is the Mellin transform of $\theta_{4}$. Finally, in Section 7, a detailed discussion is given in which we propose a number of formulas that makes the additive and multiplicative number theories have certain resemblance. Also, some open problems are given related to the the parafermionic partition functions.

\section{PARTITION FUNCTIONS AND THE EULER IDENTITY}

Here, we derive the Euler identity from the partition function of a bosonic non-interacting quantum field theory. The Euler identity says; the generating functions for the number of partitions of a given number $k$ (a positive integer) into distinct parts and the number of partitions of $k$ into odd parts are equal. Our derivation is based on the construction of the bosonic Hamiltonian of a non-interacting quantum field theory in terms of some bosonic operators and fermionic operators. This construction is close to that given by Spector [4] on partial supersymmetry. Spector [4], he assumed that the bosonic operators were given by $q_{k}=\left(b_{k}\right)^{2}$ and $q_{k}^{\dagger}=\left(b_{k}^{\dagger}\right)^{2}$, where $b_{k}^{\dagger}\left(b_{k}\right)$, are the bosonic creation(annihilation) operators respectively. However, a simple computation shows that the commutator of these operators is not a $c$-number, $\left[\left(b_{k}\right)^{2},\left(b_{m}^{\dagger}\right)^{2}\right]=\delta_{k m}\left(4 b_{k}^{\dagger} b_{k}+2\right)$. Therefore, the operators $q_{k}=\left(b_{k}\right)^{2}$ and $q_{k}^{\dagger}=\left(b_{k}^{\dagger}\right)^{2}$ are not bosonic operators. In order to motivate our decomposition of the bosonic Hamiltonian, we note that for a single bosonic mode (harmonic oscillator), the Hamiltonian is $H=\omega b^{\dagger} b, \omega$ beeing the frequency of the oscillator, then the energy may be written as $E_{n}=n \omega=2 n \omega+(2 n+1) \omega, n=0,1,2, \cdots$. Thus, the spectrum of $H$ splits into even and odd harmonics. This simple expression says that the energy $E_{n}$ may be obtained by adding to the energy of an even bosonic harmonic oscillator, the energy of a fermionic 
oscillator that has a zero frequency and a single frequency $\omega$ in its spectrum. As a consequence, the Hamiltonian of a single bosonic mode may decomposed as $H=2 \omega q^{\dagger} q+\omega c^{\dagger} c$, where $q^{\dagger}(q)$, are the bosonic creation(annihilation) operators for the even harmonic oscillator and $c^{\dagger}(c)$, are the creation (annihilation) operators that have the same effect as $b$ and $b^{\dagger}$ respectively, the difference being that they are square free (fermionic operators), that is, $(c)^{2}=\left(c^{\dagger}\right)^{2}=0$. This decomposition may extended to all the bosonic modes in the non-interacting quantum field theory. The next four sections in this paper are connected to non-interacting quantum field theories whose spectrum is not logarithmic, and so we can write the bosonic (fermionic) Hamiltonian without the logarithm of a prime in the form,

$$
\begin{aligned}
& H_{B}=\omega \sum_{k=1}^{\infty} k b_{k}^{\dagger} b_{k} . \\
& H_{F}=\omega \sum_{k=1}^{\infty} k f_{k}^{\dagger} f_{k}
\end{aligned}
$$

where $b_{k}^{\dagger}\left(b_{k}\right)$, are the bosonic creation(annihilation) operators respectively and $f_{k}^{\dagger}\left(f_{k}\right)$, are the fermionic creation (annihilation) operators respectively.the $k^{\text {th }}$ harmonic oscillator has a frequency $k \omega$. The operators $b_{k}$ and $b_{k}^{\dagger}$ satisfy the usual commutators given by $\left[b_{m}, b_{n}^{\dagger}\right]=\delta_{n m}$. The ground state $|\emptyset\rangle$ is defined by $b_{k}|\emptyset\rangle=0$, for all $k$. The quantum states denoted by $|n\rangle$ are obtained by acting with the creation operators on the ground state,i.e, $|n\rangle=\left(b_{1}^{\dagger}\right)^{n_{1}}\left(b_{2}^{\dagger}\right)^{n_{2}} \cdots\left(b_{k}^{\dagger}\right)^{n_{k}} \cdots|\emptyset\rangle$. Applying the number operator $\mathbf{n}=\sum_{k=1}^{\infty} k b_{k}^{\dagger} b_{k}$ on the state $|n\rangle$, we have $\mathbf{n}|n\rangle=n|n\rangle$ where $n=\sum_{k=1}^{\infty} k n_{k}$. For fermions, the commutators are given by $\left\{f_{k}, f_{m}^{\dagger}\right\}=\delta_{k m}$, in this case the allowed eigenvalues of the number operator $f_{k}^{\dagger} f_{k}$ are $n_{k}=1$ or 0 . Just like we did for a single mode, the bosonic Hamiltonian may be decomposed as

$$
H_{B}=\omega \sum_{k=1}^{\infty} k c_{k}^{\dagger} c_{k}+\omega \sum_{k=1}^{\infty} 2 k q_{k}^{\dagger} q_{k}
$$

where $q_{k}, q_{k}^{\dagger}$ are the bosonic creation (annihilation) operators in the even bosonic oscillators, $\left(c_{k}\right)^{2}=$ $\left(c_{k}^{\dagger}\right)^{2}=0$ are the square free operators which have the same effect as $q_{k}, q_{k}^{\dagger}$. As a result the $c_{k}$ partition function is not graded, that is,

$$
\operatorname{Tr}(-1)^{F} \exp \left(-\beta \omega \sum_{k=1}^{\infty} k c_{k}^{\dagger} c_{k}\right)=\operatorname{Tr} \exp \left(-\beta \omega \sum_{k=1}^{\infty} k c_{k}^{\dagger} c_{k}\right),
$$

where $F$ is the fermion number operator with eigenvalues 0 or 1 . It is equal to 0 on the states created by bosonic operators. The fermionic partition function may be obtained using the following identity

$$
\operatorname{Tr}\left[(-1)^{F} \exp -\beta\left(H_{B}+H_{F}\right)\right]=\operatorname{Tr} \exp -\beta H_{B} \operatorname{Tr}(-1)^{F} \exp -\beta H_{F}=1 .
$$

This identity may be checked using the expressions for $H_{B}$ and $H_{F}$, formally, this is equivalent to the Witten index [15]. Now, since the components of our Hamiltonian $H_{B}$ commute, then, the trace in the bosonic partition function decomposes as

$$
\operatorname{Tr}\left[\exp -\beta H_{B}\right]=\operatorname{Tr}\left[\exp -\beta \omega \sum_{k=1}^{\infty} k c_{k}^{\dagger} c_{k}\right] \operatorname{Tr}\left[\exp -\beta \omega \sum_{k=1}^{\infty} 2 k q_{k}^{\dagger} q_{k}\right],
$$

where the first term on the right of this equation is the fermionic partition function and the second term is the bosonic partition function. Adding the term $\omega \sum_{k=1}^{\infty} 2 k f_{k}^{\dagger} f_{k}$ to the original Hamiltonian 
and then using the identity equivalent to the Witten index, then the fermionic partition may be written as

$$
\begin{aligned}
Z_{F}(\beta) & =\operatorname{Tr}\left[\exp \left(-\beta \omega \sum_{k=1}^{\infty} k c_{k}^{\dagger} c_{k}\right)\right]=\operatorname{Tr}\left[(-1)^{F} \exp -\beta \omega \sum_{k=1}^{\infty}\left(k c_{k}^{\dagger} c_{k}+2 k q_{k}^{\dagger} q_{k}+2 k f_{k}^{\dagger} f_{k}\right)\right] \\
& =\operatorname{Tr}\left[(-1)^{F} \exp -\beta\left(H_{B}+2 H_{F}\right)\right] .
\end{aligned}
$$

The bosonic and the fermionic partition functions in equation (5) are computed in the Fock space of states for both bosons and fermions using the expressions for $H_{B}$ and $H_{F}$ given by equation (1) and equation (21) respectively, and so we have,

$$
Z_{B}(\beta)=\operatorname{Tr}\left[\exp -\beta H_{B}\right]=\operatorname{Tr}\left[\exp -\beta \omega \sum_{k=1}^{\infty} k b_{k}^{\dagger} b_{k}\right]=\prod_{k=1}^{\infty} \sum_{n_{k}=0}^{\infty} x^{n_{k} k}=\prod_{k=1}^{\infty} \frac{1}{1-x^{k}}
$$

where we have set $x=\exp -(\beta \omega)$, one can easily check that the partition function for equation (3) and that for $H_{B}$ equation (1) agree. The computation in the fermionic case is similar except that due to the Fermi-Dirac statistics $n_{k}$ takes the values 0 and 1, therefore,

$$
Z_{F}(\beta)=\operatorname{Tr}\left[\exp -\beta \omega \sum_{k=1}^{\infty} k c_{k}^{\dagger} c_{k}\right]=\prod_{k=1}^{\infty} \sum_{n_{k}=0}^{1} x^{n_{k} k}=\prod_{k=1}^{\infty}\left(1+x^{k}\right)
$$

The remaining partition function to be computed is the graded fermionic partition

$$
\operatorname{Tr}\left[(-1)^{F} \exp -\beta\left(2 H_{F}\right)\right] .
$$

Since the fermion number (the eigenvalue of $F$ ) is either 0 or 1 , then if we denote this partition by $\Delta_{F}(\beta)$ we have

$$
\Delta_{F}(2 \beta)=\operatorname{Tr}\left[(-1)^{F} \exp \left(-2 \beta \omega \sum_{k=1}^{\infty} k f_{k}^{\dagger} f_{k}\right)\right]=\prod_{k=1}^{\infty} \sum_{n_{k}=0}^{1}(-1)^{n_{k}} x^{2 n_{k} k}=\prod_{k=1}^{\infty}\left(1-x^{2 k}\right) .
$$

Finally, the factorization identity gives

$$
\prod_{k=1}^{\infty}\left(1+x^{k}\right)=\prod_{k=1}^{\infty} \frac{\left(1-x^{2 k}\right)}{\left(1-x^{k}\right)}=\prod_{k=1}^{\infty} \frac{\left(1-x^{2 k}\right)}{\left(1-x^{2 k}\right)\left(1-x^{2 k-1}\right)}=\prod_{k=1}^{\infty} \frac{1}{\left(1-x^{2 k-1}\right)} .
$$

This is a well known theorem due to Euler [5] which says; that the generating functions for the number of partitions of a given number $k$ into distinct parts and the number of partitions of $k$ into odd parts are equal. Here, we have obtained this equality from a fermionic partition function that is part of the full bosonic partition function. Next, we will write down the partition functions for the bosonic and fermionic simple harmonic oscillators respectively. These two partition functions are related to each other through the identity given by equation (5), and as a result we get a well known identity for $\cosh x$ in terms of an infinite product. The Hamiltonian for the bosonic (fermionic) simple harmonic oscillator are $H_{B}=\omega(b \dagger b+1 / 2)\left(H_{F}=\omega\left(f^{\dagger} f-1 / 2\right)\right)$ respectively 
and hence their partition functions are,

$$
\begin{aligned}
Z_{B}(\beta) & =\operatorname{Tr}\left[\exp \left(-\beta \omega\left(b^{\dagger} b+1 / 2\right)\right)\right] \\
& =\sum_{n=0}^{\infty} \exp (-\beta \omega(n+1 / 2))=\frac{1}{2 \sinh (\beta \omega / 2)} . \\
Z_{F}(\beta) & =\operatorname{Tr}\left[\exp \left(-\beta \omega\left(f^{\dagger} f-1 / 2\right)\right)\right] \\
& =\sum_{n=0}^{1} \exp [-\beta \omega(n-1 / 2)]=2(\cosh (\beta \omega / 2)),
\end{aligned}
$$

The other piece we need to compute is the graded fermionic partition function

$$
\Delta_{F}(2 \beta)=\operatorname{Tr}\left[(-1)^{F} \exp \left(-\beta \omega\left(f^{\dagger} f-1 / 2\right)\right)\right]
$$

which is simple to calculate since the eigenvalues of the fermion number operator $F$ are 0 and 1 and so we have $\Delta_{F}(2 \beta)=2 \sinh (\beta \omega / 2)$ since the infinite product representation of sine hyperbolic is $\sinh (x)=x \prod_{k=0}^{\infty}\left(1+\frac{x^{2}}{k^{2} \pi^{2}}\right)$. Then the factorization identity (5) gives

$$
\begin{aligned}
Z_{F}(\beta) & =2 \cosh (\beta \omega) / 2)=\frac{2 \sinh (\beta \omega)}{2 \sinh (\beta \omega / 2)} \\
& =2 \prod_{k=1}^{\infty} \frac{\left(1+\frac{(\beta \omega)^{2}}{k^{2} \pi^{2}}\right)}{\left(1+\frac{(\beta \omega)^{2}}{4(k)^{2} \pi^{2}}\right)}=2 \prod_{k=1}^{\infty}\left(1+\frac{(\beta \omega)^{2}}{(2 k+1)^{2} \pi^{2}}\right),
\end{aligned}
$$

which is exactly the infinite product representation of $(\cosh (\beta \omega) / 2)$, therefore, it follows that the results one obtains by writing the fermionic partition function in terms of a bosonic partition function depends very much on the Hamiltonian used. If one is dealing with a theory with a logarithmic spectrum, then this procedure gives a proof of a number theoretic identity that connects the zeta function to the modulus of Mobius inversion function [4]. Here, we gave the generating function proof of the Euler theorem in which the number of partitions of $k$ into distinct parts equals the number of partitions of $k$ into odd parts. Also, in the case the Hamiltonian is that of a harmonic oscillator, we have derived a well known identity in hyperbolic trigonome-

try $2 \cosh (\beta \omega / 2)=\frac{\sinh (\beta \omega)}{\sinh (\beta \omega / 2)}$, from which we obtain the infinite product representation of $\cosh x$ knowing that of $\sinh (x)$

\section{PARAFERMIONIC PARTITION FUNCTIONS AND PARTI- TIONS WITH RESTRICTIONS}

The natural generalization to the previous section, in which the fermionic partition function was factorized as a product of graded fermionic partition function $\Delta_{F}(2 \beta)$ times the bosonic partition function $Z_{B}(\beta)$, would be to consider parafermions of order s. As fermions are of order 2 parafermions, therefore, just like the first factorization identity (5), we will have the following second factorization identity

$$
\begin{aligned}
Z_{s}(\beta) & =\operatorname{Tr}\left[(-1)^{F} \exp -\beta\left(H_{B}+s H_{F}\right)\right], \\
& =Z_{B}(\beta) \Delta_{F}(s \beta)
\end{aligned}
$$


where the Hamiltonian $H_{B}$, is constructed out of certain operators $\chi_{k}$ and $r_{k}$ such that $\left(\chi_{k}\right)^{s}=$ $\left(\chi_{k} \dagger\right)^{s}=0$ but no lower powers vanish as operators, i.e., these are parafermionic operators, the operators $r_{k}, r_{k}^{\dagger}$ are the operators of the bosonic oscillators having frequencies with multiplicity $s$. These oscillators are the natural generalization of the even bosonic oscillators considered in the last section. Spector [4, in his construction of the bosonic Hamiltonian, he assumed that the operators given by $r_{k}=\left(b_{k}\right)^{s}, r_{k}^{\dagger}=\left(b_{k}^{\dagger}\right)^{s}$ are bosonic operators. However, from the following explicite expressions of the commutators for $s=3, s=4$,

$$
\begin{gathered}
{\left[(b)^{3},\left(b^{\dagger}\right)^{3}\right]=9 b^{\dagger} b b^{\dagger} b+9 b^{\dagger} b+6,} \\
{\left[(b)^{4},\left(b^{\dagger}\right)^{4}\right]=16 b^{\dagger} b b^{\dagger} b b^{\dagger} b+24 b^{\dagger} b b^{\dagger} b+56 b^{\dagger} b+24,}
\end{gathered}
$$

it is clear that the powers of the bosonic operators are not bosonic operators. Now, let us see how to construct the Hamiltonian of a bosonic oscillator in terms of a parafermion of order three. The energy of the bosonic oscilator in this case may be written as $E_{n}=\omega n=\omega(3 n)+\omega(3 n+$ 1) $+\omega(3 n+2), n=0,1,2, \cdots$, since both sides of this expresion give the same spectrum of the Hamiltonian $H_{B}=\omega b^{\dagger} b$. As a consequence, the Hamiltonian of a single bosonic mode may decomposed as $H_{B}=3 \omega r^{\dagger} r+\omega \chi^{\dagger} \chi$, where $r^{\dagger}(r)$, are the bosonic creation (annihilation) operators for the harmonic oscillator whose frequency is $3 \omega$, and $\chi^{\dagger}(\chi)$, are the creation (annihilation) operators that have the same effect as $b^{\dagger}(b)$ respectively, except that $\left(\chi^{\dagger}\right)^{3}=(\chi)^{3}=0$, that is, cubic free. Note that the eigenvalues of the number operator $\chi^{\dagger} \chi$ for $s=3$ are $n=0,1$, , and for general $s$, the eigenvalues are $n=0,1,2, \cdots, s-1$. In general, the decomposition of the bosonic Hamiltonian in the non-interacting quantum field theory may be witten as

$$
H_{B}=\omega \sum_{k=1}^{\infty} \chi_{k}^{\dagger} \chi_{k}+\omega \sum_{k=1}^{\infty} s r_{k}^{\dagger} r_{k}
$$

By the using the identity

$$
\operatorname{Tr}\left[(-1)^{F} \exp -\beta\left(\omega \sum_{k=1}^{\infty} s r_{k}^{\dagger} r_{k}+s \omega \sum_{k=1}^{\infty} k f_{k}^{\dagger} f_{k}\right)\right]=1,
$$

which is equivalent to the Witten index, then the parafermionic partition reads,

$$
Z_{s}(\beta)=\operatorname{Tr} \exp \left(-\beta\left(H_{s}\right)\right)=\operatorname{Tr}\left[(-1)^{F} \exp \left(-\beta\left(H_{B}+s H_{F}\right)\right)\right]
$$

where $H_{s}=\omega \sum_{k=1}^{\infty} \chi_{k}^{\dagger} \chi_{k}$. The parafermionic partition function is a sort of truncated bosonic partition since the term $x^{s k}$ and higher terms are not present and so $Z_{s}(\beta)=\prod_{k=1}^{\infty}\left(1+x^{k}+x^{2 k}+\right.$ $\left.\cdots+x^{(s-1) k}\right)$ and as a result by using the second factorization identity, we obtain

$$
\begin{aligned}
Z_{s}(\beta) & =\operatorname{Tr} \exp \left(-\beta\left(H_{s}\right)\right)=\sum_{n_{k}=0}^{s-1} x^{\sum_{k=1}^{\infty} k n_{k}} \\
& =\prod_{k=1}^{\infty}\left(1+x^{k}+x^{2 k}+\cdots+x^{(s-1) k}\right) \\
& =\prod_{k=1}^{\infty} \frac{\left(1-x^{s k}\right)}{\left(1-x^{k}\right)}
\end{aligned}
$$


This is exactly an identity in the theory of partitions [8] which says that the the generating function for the number of partitions of $k$ in which no parts occur more than $s-1$ times equals the generating function for the number of partitions of $k$ such that no parts is divisible by $s$. This is understood as we have eliminated terms of the form $\prod_{k=1}^{\infty}\left(1-x^{s k}\right)$ from the bosonic partition function which in turn is the generating function for the number of partitions of $k$ without restrictions. Therefore, this well known result in partition theory with restriction is obtained by separating the parafermionic Hamiltonians $H_{s}$, from the bosonic Hamiltonian $H_{B}$.

Next, we will give two explicit examples, the first example is connected with parafermions having non-integer order. The other example that will be considered is connected with the parabosonic of of order $s$, the partner of our parafermion of this section. We have seen in the last section that the operators $b^{2}$ and $\left(b^{\dagger}\right)^{2}$ are not bosonic operators since the commutator between these operators is not a $c$ number. In this particular situation, it is possible to write the bosonic Hamiltonian $H=\omega\left(b^{\dagger} b+\frac{1}{2}\right)$ in terms of the scaled operators $q=\frac{1}{2}(b)^{2}$ and $q^{\dagger}=\frac{1}{2}\left(b^{\dagger}\right)^{2}$, where $\left[q, q^{\dagger}\right]=b \dagger b+\frac{1}{2}$. Hence, $H=\omega\left[q, q^{\dagger}\right]$. It was shown in [6] that the operators $q, q^{\dagger}$ satisfy a modified version of the Green para-fermi commutation relations [7],

$$
\begin{gathered}
\frac{1}{2}\left[\left[q^{\dagger}, q\right], q\right]=-q, \\
{[[q, q], q]=0 .}
\end{gathered}
$$

The Fock space was constructed whose vacuum $|0\rangle$ is annihilated by $q$, and the number operator is $\mathbf{n}=\left(\frac{1}{2}\left[q, q^{\dagger}\right]-p\right)$, where $p$ is the order of the parafermion defined by $q q^{\dagger}|\emptyset\rangle=p|\emptyset\rangle$, simple computations shows that that the order of parastatistics are $p=1 / 2, p=3 / 2$. It turns out that the norm of the states in the Fock space of such infinite number of oscillators is not always positive [16]. For a single oscillator, no such difficulty appear and in this particular case, the bosonic Fock space is a direct sum of the two Fock spaces associated with parastaistics $p=1 / 2, p=3 / 2$ respectively. These Fock spaces are generated by the states

$$
|\mathbf{n}, p\rangle=\left(q^{\dagger}\right)^{n}|\mathbf{n}, \emptyset\rangle
$$

that are eigenstates of the operator

$$
\tilde{H}=\omega\left[q, q^{\dagger}\right]=\omega(2 \mathbf{n}+p)
$$

Now, the eigenstates of $H$ are $|\mathbf{n}\rangle=\left(b^{\dagger}\right)^{n}|\emptyset\rangle$, with the single vacuum $|\emptyset\rangle$, while the states of $\tilde{H}$ using the realization $q=\frac{1}{2}(b)^{2}, q^{\dagger}=\frac{1}{2}\left(b^{\dagger}\right)^{2}$ have two vacuum, $|\emptyset\rangle$ and $b^{\dagger}|\emptyset\rangle$, so that $q q^{\dagger}|\emptyset\rangle=1 / 2|\emptyset\rangle$, $q q^{\dagger} b^{\dagger}|\emptyset\rangle=3 / 2|\emptyset\rangle$. As a consequence, the states using the representation of $H$ for the two Fock spaces may be written as $|\mathbf{2 n}\rangle, n=0,1, \cdots$, and $|\mathbf{2 n}+\mathbf{1}\rangle, n=0,1, \cdots$, respectively. Next, we will write down the expressions for the partition functions in these sectors, and show that the sum of these partition functions and not the product is identical to the bosonic partition function. This follows simply by noting that the spectrum in the $p=1 / 2$ is $E_{n, 1 / 2}=(2 n+1 / 2) \omega$, while for $p=3 / 2$ is $E_{n, 1 / 2}=(2 n+1+1 / 2) \omega$. Therefore their partition functions are;

$$
Z_{1 / 2}(\beta)=\sum_{n=0}^{\infty} \exp (-\beta \omega(2 n+1 / 2))=\frac{\exp -(\beta \omega / 2)}{1-\exp -(2 \beta \omega)}
$$

and

$$
Z_{3 / 2}(\beta)=\sum_{n=0}^{\infty} \exp (-\beta \omega(2 n+1+1 / 2))=\frac{\exp -(3 \beta \omega / 2)}{1-\exp -(2 \beta \omega)}
$$


Therefore, the bosonic partition function reads,

$$
Z_{B}(\beta)=Z_{1 / 2}(\beta)+Z_{3 / 2}(\beta)=\frac{\exp -(\beta \omega)(\exp (\beta \omega / 2)+\exp -(\beta \omega / 2))}{1-\exp -(\beta \omega)}=\frac{\exp -(\beta \omega / 2)}{1-\exp -(2 \beta \omega)} .
$$

This shows clearly that

$$
Z_{B}(\beta)=Z_{1 / 2}(\beta)+Z_{3 / 2}(\beta)=Z_{B}(2 \beta) Z_{F}(\beta)
$$

that is, a bosonic system at temperature $\beta$ is equivalent to a mixture of a bosonic system whose temperature is twice as much and a fermionic system having the same temperature $\beta$. Adding the two parafermionic partition functions at the same temperature may be considered to take place in the intermediate process.

In this section, we have seen that the expression for the parafermionic partition function is $Z_{s}(\beta)=$ $\prod_{k=1}^{\infty} \frac{\left(1-x^{s k}\right)}{\left(1-x^{k}\right)}$, where $x=\exp (-\beta \omega)$, so that the energy of a given quantum state is a multiple of $\omega$. Suppose we have a quantum $s$-parafermionic gas system, that is, the maximum number of particles in a given quantum state is $s-1$, with the total energy $E=\sum_{k} n_{k} \epsilon_{k}$, where $k$ labels the one-particle energy levels $\epsilon_{k}$, and $n_{k}$ is the number of particles in state $k$. If $N=\sum_{k} n_{k}$, $N=0,1,2, \cdots$, is the total number of particles and $\mu$ is the chemical potential, Then, the grand canonical partition function may be written as

$$
Z_{F}^{s}=\prod_{k=1}^{\infty} \frac{\left(1-x_{k}^{s}\right)}{\left(1-x_{k}\right)},
$$

where $x_{k}=\exp -\beta\left(\mu-\epsilon_{k}\right)$. One may as well derive the expression of the parabosonic partition function $Z_{B}^{s}$. In the parabosonic case [17, the occupation number $n$ may take the values 0 , $s, s+1, \cdots$ and the interval of numbers $1<n<s$ is not allowed. For $s=1$, the parabosonic gas becomes a bosonic gas. Using the occupation number conditions of the parabosonic gas, then the partition function of a single parabosonic mode follows ,

$$
1+\frac{1}{1-x}-\frac{1-x^{s}}{1-x}=1+\frac{x^{s}}{1-x}
$$

therefore, the expression for parabosonic partition function is

$$
Z_{B}^{s}=\prod_{k=1}^{\infty}\left(1+\frac{x_{k}^{s}}{1-x_{k}}\right) .
$$

If one sets $s=1$ in the last formula, then the bosonic partition function is obtained which is in agreement with the parabose statistics for $s=1$. These results were quoted in [18, however, the last equation (18) differs from their expression. Let us consider a parabosonic gas in which the occupation number $n \neq 0$, and may take the values $s, s+1, \cdots$, then it is clear that the bosonic partition function is the sum of the parafermionic and parabosonic partition functions. From equation (18), the partner of the parafermionic partition function is $\prod_{k=1}^{\infty}\left(1+\frac{x^{k s}}{1-x^{k}}\right)$. The quantum states for the above statistics is characterized by specifying a number of particles in each 1-particle state $\left|n_{1}, n_{2}, \cdots\right\rangle=\left(a_{1}^{\dagger}\right)^{n_{1}}\left(a_{2}^{\dagger}\right)^{n_{2}} \cdots\left(a_{k}^{\dagger}\right)^{n_{k}} \cdots|\emptyset\rangle, n_{k} \leq s$. Here, all the operators commute among themselves. In the general case the operators do not commute and the Fock spaces of the parabosonic and the parafermionic systems are described by Green's trilinear relations [7]. Before proving some theorems in the theory of partitions using the second factorization identity, let us first consider the following simple example, take a parafermion of order three, so its partition 
function by the second factorization identity is $Z_{3}(\beta)=\prod_{k=1}^{\infty}\left(1+x^{k}+x^{2 k}\right)=\prod_{k=1}^{\infty} \frac{\left(1-x^{3 k}\right)}{\left(1-x^{k}\right)}$, the right hand side of this identity can be simplified to give

$$
Z_{3}(\beta)=\prod_{k=1}^{\infty} \frac{1}{\left(1-x^{3 k-2}\right)} \frac{1}{\left(1-x^{3 k-1}\right)}
$$

where we have used the identity $\prod_{k=1}^{\infty}\left(1-x^{3 k-2}\right)\left(1-x^{3 k-1}\right)\left(1-x^{3 k}\right)=\prod_{k=1}^{\infty}\left(1-x^{k}\right)$, the right hand of equation (19) is the number of partitions of $k$ into parts prime to 3 and so equals to the number of partitions of $k$ in which each part occurs at most two times. Using Maple for the product in equation (19) one obtain the following series

$$
1+x+2 x^{2}+\cdots+9 x^{7}+13 x^{8}+\cdots+1225 x^{30}+\cdots .
$$

In terms of partitions this means for example that the number of partition of the number 7 which are prime to 3 is 9 because

$$
\begin{gathered}
7=5+2=5+1+1=4+2+1=4+1+1+1=2+2+2+1=2+2+1+1+1= \\
2+1+1+1+1+1=1+1+1+1+1+1+1 .
\end{gathered}
$$

The sequence of partitions numbers corresponds to the coefficients in the series, therefore, this sequence is

$$
1,1,2, \cdots, 9,13, \cdots, 1225, \cdots
$$

this sequence coincides with the sequence with reference number A00726, in the On-Line encyclopedia of integer sequences [10]. Next, using the second factorization equation (15), we will give a different proof for the following three theorems. one theorem is on the Andrews' result [8] in connection with generating functions that exclude squares and the other two theorems are their generalizations [9]. The Andrews result may be stated by the following theorem;

Theorem 3.1 Let $P_{1}(n)$ be the number of partitions of $n$ in which each $k$ appears at most $k-1$ times and let $P_{2}(n)$ be the number of partitions of $n$ with no squares part. Then, $P_{1}(n)=P_{2}(n)$.

Proof. The generating function for $P_{1}(n)$ is nothing but the parafermionic partition function of order $k$, i.e.,

$$
\sum_{n=0}^{\infty} P_{1}(n) x^{n}=\prod_{k=1}^{\infty}\left(1+x^{k}+x^{2 k}+\cdots+x^{(k-1) k}\right)=\prod_{k=1}^{\infty} \frac{\left(1-x^{k^{2}}\right)}{\left(1-x^{k}\right)},
$$

the right hand side is the generating function for the number of partitions in which no square parts are present, and so equals to $\sum_{n=0}^{\infty} P_{2}(n) x^{n}$, and hence $P_{1}(n)=P_{2}(n)$. The generalization considered in [9] is to exclude polygonal numbers or $r$-gons as parts where the general polygonal number or the $k^{t h} n$-gonal number is given by $p_{k}^{n}=\frac{1}{2} k[(n-2) k-(n-4)]$, setting $n=4$, this equation gives square numbers $k^{2}$ and $n=5$ gives the $k^{\text {th }}$ - pentagonal numbers $\frac{1}{2} k(3 k-1)$, etc.

Theorem 3.2 Let $r \geq 2$ be a fixed integer. Let $P_{3}(n, r)$ be the number of partitions of $n$ in which each $k$ appears at most $(r-1)(k-1)$ times and let $P_{4}(n, r)$ be the number of partitions of $n$ where no $2 r$-gons can be used as parts. Then, $P_{3}(n, r)=P_{4}(n, r)$. 
Proof. The generating function for $P_{3}(n, r)$ is the parafermionic partition function of order $(r-$ $1)(k-1)+1$, and so we have;

$$
\sum_{n=0}^{\infty} P_{3}(n, r) x^{n}=\prod_{k=1}^{\infty}\left(1+x^{k}+x^{2 k}+\cdots+x^{k(r-1)(k-1)}\right)=\prod_{k=1}^{\infty} \frac{\left(1-x^{k[(r-1)(k-1)+1]}\right)}{\left(1-x^{k}\right)}
$$

to complete the proof we simply note that the term $k[(r-1)(k-1)+1]$ can be rewritten as $k[(r-1) k-(r-2)]=\frac{1}{2} k[(2 r-2) k-(2 r-4)]$ which is the $2 r$-gons, so the right hand side of the above equation generates partitions whose parts are free of $2 r$-gons, and hence $P_{3}(n, r)=P_{4}(n, r)$. Note that if we set $r=2$, we obtain the previous result in which no square parts are present in the partitions. The next results obtained in [9] is to exclude $2 r+1$-gons parts from partitions and is stated as follows;

Theorem 3.3 Let $P_{5}(n, r)$ be the number of partitions of $n$ in which each the part $2 k-1(k \geq 1)$ appears at most $(2 r-1)(k-1)$ times (and the frequency of even parts is unbounded). let $P_{6}(n, r)$ be the number of partitions of $n$ in which no odd-subscribed $2 r+1$-gons can be used as parts. Then, for all non-negative $n P_{5}(n, r)=P_{6}(n, r)$.

Proof. The generating function $P_{5}(n, r)$ from its definition is a product of even bosonic partition $\prod_{k=1}^{\infty} \frac{1}{\left(1-x^{2 k}\right)}$, and the odd parafermionic partition function of order $[(2 r-1)(k-1)+1]$. Therefore, similarly to the above proof we have

$$
\begin{aligned}
\sum_{n=0}^{\infty} P_{5}(n, r) x^{n} & =\prod_{k=1}^{\infty}\left(\frac{1}{1-x^{2 k}}\right)\left(1+x^{2 k-1}+x^{2(2 k-1)}+\cdots+x^{(2 k-1)(2 r-1)(k-1)}\right) \\
& =\prod_{k=1}^{\infty}\left(\frac{1}{1-x^{2 k}}\right)\left(\frac{1-x^{(2 k-1)[(2 r-1)(k-1)+1]}}{1-x^{(2 k-1)}}\right) \\
& =\prod_{k=1}^{\infty}\left(\frac{1-x^{(2 k-1)[(2 r-1)(k-1)+1]}}{1-x^{k}}\right),
\end{aligned}
$$

from the following algebraic identity

$$
\begin{aligned}
(2 k-1)[(2 r-1)(k-1)+1] & =(2 k-1)[(2 r-1) k-(2 r-2)] \\
& =\frac{(2 r+1-2)}{2}(2 k-1)^{2}-\frac{(2 r+1-4)}{2}(2 k-1),
\end{aligned}
$$

so the term $(2 k-1)[(2 r-1)(k-1)+1]$ is nothing but the $2 r+1$-gons by definition, therefore $P_{5}(n, r)=P_{6}(n, r)$.

\section{GRADED PARAFERMIONIC PARTITION FUNCTIONS}

We have already seen in section two that the graded fermionic partition function $\Delta_{F}(\beta)=$ $\operatorname{Tr}\left[(-)^{F} \exp -\beta\left(H_{F}\right)\right]=\prod_{k=1}^{\infty}\left(1-x^{k}\right)$, that is, grading is equivalent to changing the sign of $x^{k}$ in the fermionic partition function. Similarly, the graded bosonic partition function can be obtained by changing the sign of $x^{k}$ in the bosonic partition function, this follow from the fact that 
the bosonic Fock space of states may be decomposed into direct sum of the Fock space of states of even occupation number and the Fock space of states of odd occupation number. So this space is graded by the operator $(-1)^{N_{B}}$ so that $(-1)^{N_{B}}\left|n_{k}\right\rangle=(-1)^{n_{k}}\left|n_{k}\right\rangle$, that is, $(-1)^{N_{B}}$ computes the parity of the states $\left|n_{k}\right\rangle$ in the bosonic Fock space of states, it is equal to \pm 1 . Therefore, if $\Delta_{B}(\beta)$ is the graded bosonic partition function, then

$$
\begin{aligned}
\Delta_{B}(\beta) & =\operatorname{Tr}\left[(-1)^{N_{B}} \exp -\beta\left(H_{B}\right)\right] \\
& =\sum_{n_{k}=0}^{\infty}(-1)^{n_{k}} x^{\sum_{k=0}^{\infty} k n_{k}} \\
& =\prod_{k=1}^{\infty} \frac{1}{\left(1+x^{k}\right)}
\end{aligned}
$$

Note that the latter operator comes naturally here in trying to obtain the graded bosonic partition function. It was introduced in [4] in connection with the notion of the bosonic index; it is +1 for Fock space states with an even number of bosonic creation operators and -1 for Fock space states with an odd number of bosonic creation operators. Recall from the last section that the parafermion partition function is a truncation of the bosonic partition function and so the graded parafermion partition function would be the truncation of the graded bosonic partition function. There are two truncations to consider depending on the parity of the parafermion $(-1)^{s}= \pm 1$. we shall denote these partition functions by $\Delta_{s}^{ \pm}(\beta)$. Therefore, using our physical intuition, the graded parafermionic partition function is obtained from parafermionic partition function simply by changing $x^{k}$ to $-x^{k}$. Therefore, the identity in equation (16) with $x^{k}$ changed to $-x^{k}$ gives another mathematical identity,

$$
\prod_{k=1}^{\infty}\left(1-x^{k}+x^{2 k}-\cdots+(-1)^{s-1} x^{(s-1) k}\right)=\prod_{k=1}^{\infty} \frac{\left(1+(-1)^{s-1} x^{s k}\right)}{\left(1+x^{k}\right)} .
$$

Rewriting the above identity in operator form and considering separately $s$ even and odd, we end up with the following two formulae,

$$
\begin{aligned}
\Delta_{s}^{+}(\beta)= & \operatorname{Tr}\left[(-1)^{N_{S}} \exp -\beta\left(H_{s}\right)\right] \\
= & \operatorname{Tr}\left[(-1)^{N_{B}} \exp -\beta\left(H_{B}\right)\right] \operatorname{Tr}\left[(-1)^{F} \exp -\beta\left(s H_{F}\right)\right], \quad s \text { even } \\
\Delta_{s}^{-}(\beta) & =\operatorname{Tr}\left[(-1)^{N_{S}} \exp -\beta\left(H_{s}\right)\right] \\
& =\operatorname{Tr}\left[(-1)^{N_{B}} \exp -\beta\left(H_{B}\right)\right] \operatorname{Tr}\left[\exp -\beta\left(s H_{F}\right)\right], \quad s \text { odd }
\end{aligned}
$$

where the operator $(-1)^{N_{S}}$ is like $(-1)^{N_{B}}$ it is +1 for Fock space states with an even number of parafermions and -1 for Fock space states with an odd number of parafermions. The above identities are identical to equations (6.1) and (6.2) given in [4]. Next, We will seek for the physical meaning associated with the generating functions $\Delta_{s}^{ \pm}(\beta)$. For $s=2$, equation (25) gives $1 / \Delta_{2}^{+}(\beta)=\prod_{k=1}^{\infty} \frac{\left(1+x^{k}\right)}{\left(1-x^{2 k}\right)}$, which is the bosonic partition function. This can be written as $Z_{b}(\beta)=Z_{b}(2 \beta) Z_{f}(\beta)=\prod_{k=1}^{\infty} \frac{1}{\left(1-x^{k}\right)}$, that is, the partition function of the Euler gas. This partition function is known to be the generating function of partitions without restrictions; $\prod_{k=1}^{\infty} \frac{1}{\left(1-x^{k}\right)}=\sum_{k=0}^{\infty} p(k) x^{k}$, where $p(k)$ is the number of unrestricted partitions. One may obtain 
the same results by considering $s=2$ parafermion, the partition function is $\prod_{k=1}^{\infty}\left(1+x^{k}\right)$, and so the inverse of the graded parafermion partition function (IGPPF) is $1 / \Delta_{F}(\beta)=\prod_{k=1}^{\infty} \frac{1}{\left(1-x^{k}\right)}$ the bosonic partition function (infinite order parafermionic parition function). Similarly, for boson the partition function (IGPPF) is the fermionic partition function $\prod_{k=1}^{\infty}\left(1+x^{k}\right)$. Setting $s=1$, gives $1 / \Delta_{1}^{-}(\beta)=\prod_{k=1}^{\infty} \frac{\left(1+x^{k}\right)}{\left(1+x^{k}\right)}=1$, this means we have a complete cancellation of fermions. Therefore, to make connection with partition theory or physics, the generating functions that we should consider is $1 / \Delta_{s}^{ \pm}(\beta)$ depending on the parity of $s$, and so using equation (25), the partition function (s-IGPPF) may be written as

$$
\begin{aligned}
& \frac{1}{\Delta_{s}^{+}(\beta)}=Z_{B}(s \beta) Z_{F}(\beta), \\
& \frac{1}{\Delta_{s}^{-}(\beta)}=Z_{F}(\beta) \Delta_{B}(s \beta),
\end{aligned}
$$

as a consequence, $\frac{1}{\Delta_{s}^{+}(\beta)}$ for $s=4,6,8, \cdots$, is a bosonic partition function with some bosonic states missing, due to the partition function $Z_{B}(s \beta)$. Similarly, $\frac{1}{\Delta_{s}^{-}(\beta)}$ for $s=3,5,7, \cdots$, is a fermionic partition function with some fermionic states missing due to the graded bosonic partition function $\Delta_{B}(s \beta)$. For $s$ odd one may write the partition function as

$$
\frac{1}{\Delta_{s}^{-}(\beta)}=\prod_{k=1, k \nmid s}^{\infty}\left(1+x^{k}\right)=\prod_{k=1, k \nmid s}^{\infty} \frac{1}{\left(1-x^{2 k-1}\right)}
$$

For example, consider a non-relativistic bosonic quantum string [19] whose Hamiltonian $H_{B}=$ $\omega \sum_{k=1}^{\infty} k a_{k}^{\dagger} a_{k}$ that is, the $k^{t h}$ harmonic oscillator has a frequency $k \omega$. The operators $a_{k}$ and $a_{k}^{\dagger}$ satisfy the usual commutators given by $\left[a_{m}, a_{n}^{\dagger}\right]=\delta_{n m}$. The ground state $|\emptyset\rangle$ is defined by $a_{k}|\emptyset\rangle=0$, for all $k$. The quantum states of the string denoted by $|n\rangle$ are obtained by acting with the creation operators on the ground state,i.e, $|n\rangle=\left(a_{1}^{\dagger}\right)^{n_{1}}\left(a_{2}^{\dagger}\right)^{n_{2}} \cdots+\left(a_{k}^{\dagger}\right)^{n_{k}} \cdots|\emptyset\rangle$. Applying the number operator $\mathbf{n}=\sum_{k=1}^{\infty} k a_{k}^{\dagger} a_{k}$ on the state $|n\rangle$, we have $\mathbf{n}|n\rangle=n|n\rangle$ where $n=\sum_{k=1}^{\infty} k n_{k}$. One can see that for a fixed $n$, the solutions to the latter equation are given by $p(n)$. Therefore $p(n)$ counts the number of states with a given eigenvalue $n$. for example $n=3, p(3)=3$ since the partitions of 3 are; $3,2+1$, and $1+1+1$. In terms of quantum states with a number eigenvalue 3 , we have 3 states which are $a_{3}^{\dagger}|\emptyset\rangle, a_{2}^{\dagger} a_{1}^{\dagger}|\emptyset\rangle$ and $\left(a_{1}^{\dagger}\right)^{3}|\emptyset\rangle$. Therefore, a Quantum state is constructed by attaching each part of the partitions a subscript to an oscillator $a^{\dagger}$. In the same way one may consider a fermionic non-relativistic quantum string whose Hamiltonian is $H_{F}=\omega \sum_{k=1}^{\infty} k \psi_{k}^{\dagger} \psi_{k}$ with $\left\{\psi_{m}, \psi_{n}^{\dagger}\right\}=\delta_{n m}$. The same construction follows for the quantum fermionic states in which we attach each part of the partitions to an oscillator $\psi^{\dagger}$, with the constraint that the configuration numbers, $n_{k}=1$ or 0 . Note that the Euler theorem in terms of quantum states is equivalent to say for a fixed eigenvalue number $n$, the number of fermionic states is equal to the number of bosonic states obtained from bosonic creation operators with odd subscripts, and with the same eigenvalue number $n$. Therefore, fermionic states are parts of the bosonic states in the Euler gas. Now, let us consider the graded parafermion of order three, its partition function is 


$$
\begin{aligned}
\Delta_{3}^{-}(\beta)=\prod_{k=1}^{\infty}\left(1-x^{k}+x^{2 k}\right)=\prod_{k=1}^{\infty} & \frac{\left(1+x^{3 k}\right)}{\left(1+x^{k}\right)}, \text { or equivalently } \\
\Delta_{3}^{-}(\beta) & =\prod_{k=1}^{\infty}\left(1-x^{k}+x^{2 k}\right) \\
& =\prod_{k=1}^{\infty} \frac{\left(1+x^{3 k}\right)}{\left(1+x^{k}\right)} \\
& =\prod_{k=1}^{\infty} \frac{1}{\left(1+x^{3 k-2}\right)} \frac{1}{\left(1+x^{3 k-1}\right)} .
\end{aligned}
$$

Then the partition function (3-IGPPF) may be written as

$$
\begin{aligned}
\frac{1}{\Delta_{3}^{-}(\beta)} & =\prod_{k=1}^{\infty}\left(1+x^{3 k-2}\right)\left(1+x^{3 k-1}\right) \\
& =\prod_{k=1}^{\infty} \frac{\left(1-x^{3(2 k-1)}\right)}{\left(1-x^{2 k-1}\right)} \\
& =\prod_{k=1}^{\infty} \frac{1}{\left(1-x^{6 k-5}\right)} \frac{1}{\left(1-x^{6 k-1}\right)},
\end{aligned}
$$

where the first line of the above equation is the generating function of the number of partitions of $k$ into distinct parts which are prime to 3 . The last line On the hand represents the generating function for the number of partitions of $k$ into parts congruent to 1 or 5 modulo 6 . This was proved by I. Schur In 1926 [20]. Thus, we learn that the partition function (3-IGPPF) coincides with the generating function for the numbers of partitions in the Schur's theorem. In terms of quantum states, the only fermionic operators $\psi_{k}^{\dagger}$ that we consider are those for which $k$ is congruent to 1,2 modulo 3 . When $s=4$, then, the partition function (4-IGPPF) is

$$
\begin{aligned}
\frac{1}{\Delta_{4}^{+}(\beta)} & =\prod_{k=1}^{\infty} \frac{\left(1+x^{k}\right)}{\left(1-x^{2(2 k)}\right)} \\
& =\prod_{k=1}^{\infty} \frac{\left(1+x^{2 k-1}\right)}{\left(1-x^{2 k}\right)}
\end{aligned}
$$

where the the first product on the right hand side of this equation gives the number of partitions of $k$ in which each even part occurs with even multiplicity. There is no restriction on the odd parts. This generating function corresponds to sequence A006950 [10]. It is also the number of partitions of $k$ in which all odd parts occur with multiplicity one. There is no restriction on the even parts. This was a comment that we made on this sequence [10. In terms of quantum states, equation (32), tells us that these states are constructed such that the operators $\left(a_{k}^{\dagger}\right), k$ is a multiple of 2 , appear with even multiplicity. There is no restriction on those operators whose subscripts are odd. For example the number of partitions of 4 is 3 because 4 can be written as $2+2,3+1$ and $1+1+1+1$. The corresponding quantum states are $a_{2}^{\dagger^{2}}|\emptyset\rangle, a_{3}^{\dagger} a_{1}^{\dagger}|\emptyset\rangle$ and $\left(a_{1}^{\dagger}\right)^{4}|\emptyset\rangle$. It was shown in [21], that the partition function given in equation (32), is related to the irreducible characters $\chi_{0}(q)$ and $\chi_{1 / 2}(q)$ of the $c=1 / 2$ Virasoro algebra. More explicitly, the coefficients in

$$
q^{-1 / 16} \frac{\chi_{0}(q)}{\eta}=q^{-1 / 16} \sum_{n=0}^{\infty} b_{n} q^{n}=q^{-1 / 16}\left(1+q+3 q^{2}+5 q^{3}+10 q^{4}+\cdots\right),
$$


and

$$
q^{7 / 16} \frac{\chi_{1 / 2}(q)}{\eta}=q^{7 / 16} \sum_{n=0}^{\infty} c_{n} q^{n}=q^{71 / 16}\left(1+2 q+4 q^{2}+7 q^{3}+13 q^{4}+\cdots\right),
$$

are given by $b_{n}=a_{2 n}, c_{n}=a_{2 n+1}$, respectively, where $a_{n}$ is the expansion coefficient of $\frac{1}{\Delta_{4}^{+}(\beta)}$. As a consequence, the coefficients $b_{n}, c_{n}$ may be given interpretation in terms of partitions, this follows easily from the above definitions and it is not difficult to see that $b_{n}$ is the number of partitions of $2 n$, such that even part occurs with even multiplicity. There is no restriction on the odd parts. Alternatively, the number of partitions of $2 n$ in which all odd parts occur with multiplicity one. There is no restriction on the even parts. The same definition is given to $c_{n}$, but for the partition of the number $2 n+1$. Very recently, the coefficients of the partition function (4-IGPPF), are associated with the counting of the Moore-Read edge spectra in the anti-periodic sectors [14]. The next generating functions to consider, are the partition functions (5-IGPPF) and (6-IGPPF) respectively,

$$
\begin{aligned}
\frac{1}{\Delta_{5}^{+}(\beta)} & =\prod_{k=1}^{\infty} \frac{\left(1+x^{k}\right)}{\left(1+x^{5 k}\right)} \\
& =\prod_{k=1}^{\infty}\left(1+x^{5 k-1}\right)\left(1+x^{5 k-2}\right)\left(1+x^{5 k-3}\right)\left(1+x^{5 k-4}\right) \\
& =\prod_{k=1}^{\infty} \frac{1}{\left(1-x^{10 k-1}\right)\left(1-x^{10 k-3}\right)\left(1-x^{10 k-7}\right)\left(1-x^{10 k-9}\right)} \\
\frac{1}{\Delta_{6}^{+}(\beta)} & =\prod_{k=1}^{\infty} \frac{\left(1+x^{k}\right)}{\left(1-x^{3(2 k)}\right)} \\
& =\prod_{k=1}^{\infty} \frac{\left(1+x^{3 k-1}\right)\left(1+x^{3 k-2}\right)}{\left(1-x^{3 k}\right)} \\
& =\prod_{k=1}^{\infty} \frac{1}{\left(1-x^{6 k-1}\right)\left(1-x^{6 k-3}\right)\left(1+x^{6 k-5}\right)\left(1-x^{6 k}\right)} .
\end{aligned}
$$

Equation (33) gives the number of partitions of $k$ into distinct parts prime to 5 . Equivalently, It is the number of partitions of $k$ into parts congruent to 1,3,7,9 modulo 10. This generalizes parts of Schur's theorem. The second definition is seen simply by applying the Euler theorem to the fourth term on the right hand side of equation (33), then write each denominator as a product of even and odd parts. The first term of the right hand side of equation (33), gives the number of partitions of $k$ in which each even part occurs with a multiple of 3 . There is no restriction on the odd parts. From the second term of equation (33), it is also the number of partitions into parts congruent to $0,1,3,5$ modulo 6 . The generating functions $\frac{1}{\Delta_{5}^{-}(\beta)}, \frac{1}{\Delta_{6}^{+}(\beta)}$ correspond to sequences by the author A096938 and A096981 respectively, [10]. Let us now consider some examples associated with the partition functions $1 / \Delta_{s}^{ \pm}(\beta)$. Using Maple, one could generate sequences of partitions from our formulae depending on $s$. When $s=3$ we have,

$$
1+x+x^{2}+\cdots+3 x^{7}+3 x^{8}+3 x^{9}+4 x^{10}+\cdots
$$


so for example the number of distinct partitions of the number 10 in which the parts are prime to 3 is 4 (which we denote by $a(10)=4$ ) because $10=8+2=7+2+1=5+4+1$. The corresponding sequence of partitions is,

$$
1,1,1,1,1,2,2,3,3,3,4,5,6,7,8,9,10,12, \cdots
$$

this is the sequence $A 003105$ on the on-Line Encyclopedia of integer sequences [10]. From Schur theorem the number of partitions 10 prime to 3 is equal to the number of partitions of 10 congruent to 1,5 modulo 10 . This is so, as 10 can be written as $7+1+1+1=5+5=5+1+1+1+1+1=$ $1+1+1+1+1+1+1+1+1+1$. The second part of Schur's theorem tells us that both of these number of partitions are equal to the partition of 10 into parts that differs by at least 3 . This is indeed the case, because we can write 10 as, $10=9+1=8+2=7+3$. In terms of quantum states of the quantum string, the number of fermionic states in which the subscripts of the fermionic operators are congruent to 1,2 modulo 3 , equals the number of bosonic states in which the subscripts of the bosonic operators are congruent to 1,5 modulo 6 . The next sequence of partitions of the number $k$ would be that which corresponds to $s=4$, which is,

$$
1,1,1,2,3,4,5,7,10,13,16,21, \cdots
$$

this is also in [10] and corresponds to the sequence A006950. It was pointed out by Sellers, that the number of partitions for this sequence that we mentioned above for $s=4$, it is also the number of partitions of $k$ into parts not congruent to $2 \bmod 4$. therefore for $s=4$, we have three definitions. For example, the number of partition of 7 equals $7, a(7)=7$ because

$7=5+1+1=3+3+1=3+1+1+1+1=3+2+2=2+2+1+1+1=1+1+1+1+1+1+1$,

$$
7=6+1=5+2=4+3=4+2+1=3+2+2=2+2+2+1 .
$$

or

$7=5+1+1=4+3=4+1+1+1=3+3+1=3+1+1+1+1=1+1+1+1+1+1+1$.

The fifth and the sixth orders from our formulae for the inverted parafermionic partition function give the following sequences of partition of the number $k$,

$$
1,1,1,2,2,2,3,4,4,6,7,8,10,16.19,22,26,30,35,41, \cdots
$$

for the fifth order and

$$
1,1,1,2,2,3,5,6,7,10,12,15,25,30,39,46, \cdots
$$

for the sixth order respectively. In the fifth order, of the inverted parafermionic partition function, the number 5 and its multiples are not present in the partitions of $k$ into distinct parts, for example $9=8+1=7+2=6+3=6+2+1=4+3+2$ so $a(9)=6$. From the second definition for $s=5$, this also the number of partition of 9 into parts congruent to 1,3,7,9 modulo 10. This is indeed the case, since $9=7+1+1=3+3+3=3+3+1+1+1=3+1+1+1+1+1+1=1+1+1+1+1+1+1+1+1$. For the sixth order sequence the number of partitions of the number 11 for example, is $15, a(11)=15$. Because 
$11=9+1+1=7+3+1=7+1+1+1+1=5+5+1=5+3+3=5+3+1+1+1=5+2+2+2=$ $5+1+1+1+1+1+1=3+3+3+1+1=3+3+1+1+1+1+1=3+2+2+2+1+1=$ $3+1+1+1+1+1+1+1+1=2+2+2+1+1+1+1+1=1+1+1+1+1+1+1+1+1+1+1$.

This is also given by the number of partitions of 11 congruent to $0,1,3,5$ modulo 6 . Because

$$
\begin{gathered}
11=9+1+1=7+3+1=7+1+1+1+1=6+5=6+3+1+1=6+1+1+1+1+1= \\
5+5+1=5+3+3=5+3+1+1+1=5+1+1+1+1+1+1=3+3+3+1+1= \\
3+3+1+1+1+1+1=3+1+1+1+1+1+1+1+1=1+1+1+1+1+1+1+1+1+1+1 .
\end{gathered}
$$

Therefore, we see that our generating function gives known sequences for partitions of integers as well as new ones. In terms of partition functions, when $s$ is even then, the generating function $\frac{1}{\Delta_{s}^{+}(\beta)}$ is definitely bosonic partition with some restrictions. This partition function is identical to an other bosonic partition function with different restrictions. When $s$ is odd, then, the generating function $\frac{1}{\Delta_{s}^{-}(\beta)}$ is a fermionic partition function, for for $s=1$, we have a complete cancelation of fermions. One should note, however, that the fermionic partition function is identical to a bosonic partition function with different restrictions, i.e, we have a fermi-bose equivalence. From the explicit examples given above for $s=3,4,5$ and 6 , one can see that the definitions for $\frac{1}{\Delta_{s}^{ \pm}(\beta)}$ can be generalized for any $s$, and are summarized by the following proposition.

Proposition 4.1 The bosonic partition function $\frac{1}{\Delta_{s}^{+}(\beta)}$, is the generating function for partitions of $k$ in which all odd parts are unrestricted. The even parts occur with multiples of s/2. Alternatively it is the number of partitions of $k$ into parts congruent to $0,1,3,5, \cdots,(s-1)$ modulo $s$. The fermionic partition function, $\frac{1}{\Delta_{s}^{-}(\beta)}$, is the generating function for partitions of $k$ into distinct parts prime to $s$. This is also the generating function for partitions of $k$ into parts congruent to $1,3,5, \cdots,(\hat{s}) \cdots,(2 s-1)$ modulo $(2 s)$. Note that $\hat{s}$ means that the parts are not congruent to $s$ modulo $(2 s)$.

Proof:

$$
\begin{aligned}
\frac{1}{\Delta_{s}^{+}(\beta)} & =\prod_{k=1}^{\infty} \frac{\left(1+x^{k}\right)}{\left(1-x^{s k}\right)} \\
& =\prod_{k=1}^{\infty} \frac{1}{\left(1-x^{2 k-1}\right)\left(1-x^{s / 2(2 k)}\right)} \\
& =\prod_{k=1}^{\infty} \frac{1}{\left(1-x^{s k}\right)\left(1-x^{s k-1}\right)\left(1-x^{s k-3}\right) \cdots\left(1-x^{s k-(s-1)}\right)},
\end{aligned}
$$

where the last line, may be obtained from the following identity

$$
\prod_{k=1}^{\infty}\left(1-x^{2 k-1}\right)=\prod_{k=1}^{\infty}\left(1-x^{s k-1}\right)\left(1-x^{s k-3}\right) \cdots\left(1-x^{s k-(s-1)}\right)
$$


for $s$ even. This proves the first part of the proposition. Using the same identity for $s$ odd, and change $s$ to $2 s$ and the Euler identity, then

$$
\begin{aligned}
\frac{1}{\Delta_{s}^{-}(\beta)} & =\prod_{k=1}^{\infty} \frac{\left(1+x^{k}\right)}{\left(1+x^{s k}\right)} \\
& =\prod_{k=1}^{\infty} \frac{1}{\left(1-x^{2 k-1}\right)\left(1+x^{s k}\right)} \\
& =\prod_{k=1}^{\infty} \frac{\left(1-x^{2 s k-s}\right)}{\left(1-x^{2 s k-1}\right)\left(1-x^{2 s k-3}\right) \cdots\left(1-x^{2 s k-s}\right) \cdots\left(1-x^{2 s k-(2 s-1)}\right)}
\end{aligned}
$$

the last identity shows that the fermionic partition is indeed the generating function for partitions of $k$ into parts congruent to $1,3,5, \cdots,(\hat{s}) \cdots,(2 s-1)$ modulo $(2 s)$. Being a generating function of partitions into distinct parts prime to $s$ is obvious from the first identity. Before finishing this section, we would like to point out that the generating functions $\frac{1}{\Delta_{s}^{ \pm}(\beta)}$ may be considered as truncation of fermions. They are the analogue of of truncation of bosons. Explicitly,

$$
\begin{aligned}
\frac{1}{\Delta_{s}^{+}(\beta)} & =\prod_{k=1}^{\infty} \frac{\left(1+x^{k}\right)}{\left(1-x^{(s / 2) k)}\right)\left(1+x^{(s / 2) k}\right)} \\
& =\prod_{k=1, k \nmid s / 2}^{\infty}\left(1+x^{k}\right) \prod_{k=1}^{\infty} \frac{1}{\left(1-x^{(s / 2) k}\right)}
\end{aligned}
$$

where the notation $k \nmid s / 2$, means that $k$ is not divisible by $s / 2$. Here, we have a sort of exchange between fermions and bosons, that is, those fermion missing from the spectrum are replaced by bosons. Therefore for $s$ even, the only fermionic operators that appear in the construction of the fermionic states, are $\psi_{k}^{\dagger}$ for which $k$ is not divisible by $s / 2$. For the bosonic operators, however, $a_{k}^{\dagger}$ are those for which $k$ is a multiple of $s / 2$. when $s$ is odd we have a fermionic truncation,

$$
\begin{aligned}
\frac{1}{\Delta_{s}^{-}(\beta)} & =\prod_{k=1}^{\infty} \frac{\left(1+x^{k}\right)}{\left(1+x^{s k}\right)} \\
& =\prod_{k=1, k \nmid s}^{\infty}\left(1+x^{k}\right) .
\end{aligned}
$$

\section{GENERATING FUNCTIONS AND THE JACOBI THETA- FUNCTION, $\theta_{4}(0, x)$}

In this section, we will construct partition functions from the parafermionic partition function and the inverse graded parafermion partition function (s-IGPPF). We will find that our partition functions are connected with the Jacobi theta function $\theta_{4}(0, x)$, and also find some sequences in partition theory. These partition functions corresponding to two different systems, namely, either by mixing parafermionic system of order $s$ with a system whose partition function is $\frac{1}{\Delta_{s}^{+}(\beta)}$ or $\frac{1}{\Delta_{s}^{-}(\beta)}$. Therefore, using the identities given in equations (16), (28), and (29) we obtain the 
following two partition functions;

$$
\begin{aligned}
\frac{Z_{s}(\beta)}{\Delta_{s}^{+}(\beta)} & =Z_{F}(\beta) Z_{B}(\beta) \\
& =\frac{\prod_{k=1}^{\infty}\left(1+x^{k}+x^{2 k}+\cdots+x^{(s-1) k}\right)}{\prod_{k=1}^{\infty}\left(1-x^{k}+x^{2 k}-\cdots-x^{(s-1) k}\right)} \\
& =\prod_{k=1}^{\infty} \frac{\left(1+x^{k}\right)}{\left(1-x^{k}\right)}, \\
\frac{Z_{s}(\beta)}{\Delta_{s}^{-}(\beta)} & =Z_{F}(\beta) Z_{B}(\beta) \Delta_{B}(s \beta) \Delta_{F}(s \beta) \\
& =\frac{\prod_{k=1}^{\infty}\left(1+x^{k}+x^{2 k}+\cdots+x^{(s-1) k}\right)}{\prod_{k=1}^{\infty}\left(1-x^{k}+x^{2 k}-\cdots+x^{(s-1) k}\right)} \\
& =\prod_{k=1}^{\infty} \frac{\left(1+x^{k}\right)}{\left(1-x^{k}\right)} \frac{\left(1-x^{s k}\right)}{\left(1+x^{s k}\right)} .
\end{aligned}
$$

Here, we would like to make some remarks about the above identities and their implications. The first identity shows that for $s$ even, mixing a parafermionic system of order $s$ with a system whose partition function is $\frac{1}{\Delta_{s}^{+}(\beta)}$, is identical to a mixed system of bosons and fermions. Note the independence on the parameter $s$ of the partition function of the mixed system in this case. This follows simply from the the cancellation of the expression $Z_{B}(s \beta)$ from the partition functions $Z_{s}(\beta)$ and $\frac{1}{\Delta_{s}^{+}(\beta)}$. Therefore, for $s$ even, the same expression for the total partition function of the mixed system may be obtained by letting the order $s$ in both the parafermion partition function and the partition function (s-IGPPF) to go to infinity. For $s$ odd, there are partial cancellations between $Z_{F}$ and $\Delta_{B}(s \beta)$, similarly between $Z_{B}(\beta)$ and $\Delta_{F}(s \beta)$. Due to the cancellations of bosons and fermions in this case, we may as well write equation (40) in the form

$$
\frac{Z_{s}(\beta)}{\Delta_{s}^{-}(\beta)}=\prod_{k=1, k \nmid s}^{\infty} \frac{\left(1+x^{k}\right)}{\left(1-x^{k}\right)}
$$

Mathematically speaking, for $s$ even the ratio of the parafermionic partition function to the graded parafermionic partition function is always given by $\prod_{k=1}^{\infty} \frac{\left(1+x^{k}\right)}{\left(1-x^{k}\right)}$. This product may be written as a sum if we set $a=1$ in the following formula [11]

$$
\sum_{n=0}^{\infty} \frac{(-a ; x)_{n}}{(x ; x)_{n}} x^{n}=\frac{(-a x ; x)_{\infty}}{(x ; x)_{\infty}},
$$

where $(a ; x)_{n}:=(1-a)(1-a x) \cdots\left(1-a x^{n-1}\right),(a ; x)_{0}=1$ and $(a ; x)_{\infty}:=\prod_{n=0}^{\infty}\left(1-a x^{n}\right)$, to obtain

$$
\begin{aligned}
\frac{Z_{s}(\beta)}{\Delta_{s}^{+}(\beta)} & =\prod_{k=1}^{\infty} \frac{\left(1+x^{k}\right)}{\left(1-x^{k}\right)} \\
& =1+\sum_{k=1}^{\infty} \frac{2(1+x)\left(1+x^{2}\right) \cdots\left(1+x^{k-1}\right)}{(1-x)\left(1-x^{2}\right) \cdots\left(1-x^{k}\right)} x^{k}
\end{aligned}
$$


This is known to be equal to the inverse of $\theta_{4}(0, x)=\sum_{n=-\infty}^{\infty}(-1)^{n} x^{n^{2}}$ via Gauss's identity. The second identity, in the case of $s$ odd, does not give $\frac{1}{\theta_{4}(0, x)}$ but rather the ratio $\theta_{4}\left(0, x^{s}\right) / \theta_{4}(0, x)$, so this extra factor can be thought of as correction factor that one has to insert in $\frac{Z_{s}(\beta)}{\Delta_{s}^{+}(\beta)}$. Now, rewriting our partition functions in terms of $\theta_{4}(0, x)$, then we obtain;

$$
\begin{aligned}
\frac{Z_{s}(\beta)}{\Delta_{s}^{+}(\beta)} & =\prod_{k=1}^{\infty} \frac{\left(1+x^{k}\right)}{\left(1-x^{k}\right)} \\
& =\frac{1}{\theta_{4}(0, x)}, s \text { even } \\
\frac{Z_{s}(\beta)}{\Delta_{s}^{-}(\beta)} & =\prod_{k=1}^{\infty} \frac{\left(1+x^{k}\right)}{\left(1-x^{k}\right)} \frac{\left(1-x^{s k}\right)}{\left(1+x^{s k}\right)} \\
& =\frac{\theta_{4}\left(0, x^{s}\right)}{\theta_{4}(0, x)}, s \text { odd }
\end{aligned}
$$

mixing a parafermionic system of order $s$ with a system whose partition function, $\frac{1}{\Delta_{s}^{ \pm}(\beta)}$, we produce two systems whose partition functions are $\frac{1}{\theta_{4}(0, x)}$ and $\frac{\theta_{4}\left(0, x^{s}\right)}{\theta_{4}(0, x)}$ respectively. The second partition function is obtained from the first one by canceling some of the states from the full Fock space of bosons and fermions. Note that the partition $\frac{1}{\theta_{4}(0, x)}$, is also the partition function for the free theory formed by a single holomorphic boson and holomorphic fermion with anti-periodic spin structure on the torus [21]. In terms of partition theory, and referring to sequence A015128 in [10], $\frac{1}{\theta_{4}(0, x)}$ is known to be generating function for partitions of $2 k$ with all odd parts occurring with even multiplicities. There is no restriction on the even parts. The proof is straightforward, and follows from the Euler theorem;

$$
\begin{aligned}
\frac{1}{\theta_{4}(0, x)} & =\prod_{k=1}^{\infty} \frac{\left(1+x^{k}\right)}{\left(1-x^{k}\right)} \\
& =\prod_{k=1}^{\infty} \frac{1}{\left(1-x^{(2 k-1)}\right)\left(1-x^{k}\right)}
\end{aligned}
$$

then by changing the variable $x$ to $x^{2}$, and using equation (37), it follows that the expansion coefficients of $\frac{1}{\theta_{4}\left(0, x^{2}\right)}$ are identical to those of $\frac{1}{\theta_{4}(0, x)}$. Therefore, the generating function $\frac{1}{\theta_{4}\left(0, x^{2}\right)}=\prod_{k=1}^{\infty} \frac{1}{\left(1-x^{2(2 k-1)}\right)\left(1-x^{2 k}\right)}$ says that; the odd parts occur with even multiplicities. There is no restriction on the even parts. This is the definition we have given to the sequence A015128. Using Maple, the sequence for $\frac{1}{\theta_{4}(0, x)}$ is,

$$
1,2,4,8,14,24,40,64,100,154, \cdots, 9904,13288, \cdots \text {. }
$$

This sequence also appear in the counting of the Moore-read edge spectra together with the sequence generated by the partition function (4-IGPPF), however, the above sequence is associated with a periodic sector [14]. For example, the number of partitions of 6 is 8 . This is because, we can write 6 as

$6=4+2=4+1+1=3+3=2+2+2=2+2+1+1=2+1+1+1+1=1+1+1+1+1+1$. 
In terms of quantum states, for a fixed number eigenvalue $n$, the number of states obtained from the total partition function given by equation (42) should be equal to the number of bosonic states with a fixed number eigenvalue $2 n$. The states are constructed by applying creation operators $a_{k}^{\dagger}$ on the ground state whose subscripts are the parts of the partitions of $2 n$. We can check this for the above example as follows, the Fock space of states corresponding to the partition function given by equation (42) is a tensor product of the fermionic and bosonic Fock spaces. The bosonic Fock space of states having a number eigenvalue up to 3 are;

$$
a_{3}^{\dagger}|\emptyset\rangle, a_{2}^{\dagger} a_{1}^{\dagger}|\emptyset\rangle, a_{2}^{\dagger}|\emptyset\rangle, a_{1}^{\dagger^{2}}|\emptyset\rangle, a_{1}^{\dagger}|\emptyset\rangle, a_{1}^{\dagger^{3}}|\emptyset\rangle,|\emptyset\rangle .
$$

For the fermionic Fock space of states whose ground state is $|\tilde{\emptyset}\rangle$, we have

$$
\psi_{3}^{\dagger}|\tilde{\emptyset}\rangle, \psi_{2}^{\dagger} \psi_{1}^{\dagger}|\tilde{\emptyset}\rangle,|\tilde{\emptyset}\rangle, \psi_{2}^{\dagger}|\tilde{\emptyset}\rangle, \psi_{1}^{\dagger}|\tilde{\emptyset}\rangle,|\tilde{\emptyset}\rangle
$$

Now taking the tensor product of these states such that $n=3$, we obtain the following states;

$\psi_{3}^{\dagger}|\tilde{\emptyset}\rangle \otimes|\emptyset\rangle, \psi_{2}^{\dagger} \psi_{1}^{\dagger}|\tilde{\emptyset}\rangle \otimes|\emptyset\rangle, \psi_{2}^{\dagger}|\tilde{\emptyset}\rangle \otimes a_{1}^{\dagger}|\emptyset\rangle, \psi_{1}^{\dagger}|\tilde{\emptyset}\rangle \otimes a_{2}^{\dagger}|\emptyset\rangle, \psi_{1}^{\dagger}|\tilde{\emptyset}\rangle \otimes a_{1}^{\dagger^{2}}|\emptyset\rangle,|\tilde{\emptyset}\rangle \otimes a_{3}^{\dagger}|\emptyset\rangle,|\tilde{\emptyset}\rangle \otimes a_{2}^{\dagger} a_{1}^{\dagger}|\emptyset\rangle,|\tilde{\emptyset}\rangle \otimes a_{1}^{\dagger^{3}}|\emptyset\rangle$.

These are exactly the number of partitions of 6 . We have seen that mixing a parafermionic system of an even order, with a system whose partition function (s-IGPPF) having the same order gives a bosonic system and has a number eigenvalue $2 n$. These states for the number eigenvalue 6 , are;

$$
a_{6}^{\dagger}|\emptyset\rangle, a_{4}^{\dagger} a_{2}^{\dagger}|\emptyset\rangle, a_{4}^{\dagger} a_{1}^{\dagger^{2}}|\emptyset\rangle a_{3}^{\dagger^{2}}|\emptyset\rangle, a_{2}^{\dagger^{3}}|\emptyset\rangle, a_{2}^{\dagger^{2}} a_{1}^{\dagger^{2}}|\emptyset\rangle, a_{2}^{\dagger} a_{1}^{\dagger^{4}}|\emptyset\rangle, a_{1}^{\dagger^{6}}|\emptyset\rangle
$$

In the case of a system whose partition function is $\frac{\theta_{4}\left(0, x^{s}\right)}{\theta_{4}(0, x)}$ for $s$ odd, it turns out that this particular partition function generates sequences in partition theory. As an example setting $s=3$ in equation (43), then we have

$$
\frac{Z_{3}(\beta)}{\Delta_{3}^{-}(\beta)}=\frac{\theta_{4}\left(0, x^{3}\right)}{\theta_{4}(0, x)}
$$

from the product-sum identity 6 in Slater list on the Rogers-Ramanujan type identities [22], corrected by Sills [23] in a Ph.D thesis, we learn that our partition function can be written by the following explicit sum;

$$
\begin{aligned}
\frac{\theta_{4}\left(0, x^{3}\right)}{\theta_{4}(0, x)} & =\prod_{k=1}^{\infty} \frac{\left(1+x^{3 k-1}\right)\left(1+x^{3 k-2}\right)}{\left(1-x^{3 k-1}\right)\left(1-x^{3 k-2}\right)} \\
& =1+\sum_{k=1}^{\infty} \frac{2(1+x)\left(1+x^{2}\right) \cdots\left(1+x^{k-1}\right)}{(1-x)\left(1-x^{2}\right) \cdots\left(1-x^{k}\right)(1-x)\left(1-x^{3}\right) \cdots\left(1-x^{2 k-1}\right)} x^{k^{2}} .
\end{aligned}
$$

Using Maple the sequence corresponding to $\frac{\theta_{4}\left(0, x^{3}\right)}{\theta_{4}(0, x)}$ is,

$$
1,2,4,6,10,16,24,36,52,74,104,144,198,268,360, \cdots, 48672,59122, \cdots \text {. }
$$

This corresponds to the number of partitions of $2 k$ prime to 3 with all odd parts occurring with even multiplicities. There is no restriction on the even parts e.g $a(6)=6$ because we can write 6 as, 


$$
4+2=4+1+1=2+2+2=2+2+1+1=2+1+1+1+1=1+1+1+1+1+1 .
$$

This sequence has reference number A098151 on the on-Line Encyclopedia of integer sequences [10]. This can be proved as follows;

$$
\begin{aligned}
\frac{\theta_{4}\left(0, x^{3}\right)}{\theta_{4}(0, x)} & =\prod_{k=1}^{\infty} \frac{\left(1+x^{k}\right)}{\left(1-x^{k}\right)} \frac{\left(1-x^{3 k}\right)}{\left(1+x^{3 k}\right)} \\
& =\prod_{k=1}^{\infty} \frac{\left(1-x^{3 k}\right)}{\left(1-x^{k}\right)} \frac{\left(1-x^{6 k-3}\right)}{\left(1-x^{(2 k-1)}\right)}
\end{aligned}
$$

where we have used the Euler's theorem in obtaining the last identity. Next changing $x$ to $x^{2}$, then we have,

$$
\begin{aligned}
\frac{\theta_{4}\left(0, x^{2(3)}\right)}{\theta_{4}\left(0, x^{2}\right)} & =\prod_{k=1}^{\infty} \frac{\left(1-x^{2(3 k)}\right)}{\left(1-x^{2 k}\right)} \frac{\left(1-x^{2.3(2 k-1)}\right)}{\left(1-x^{2(2 k-1)}\right)} \\
& =\prod_{k=1}^{\infty} \frac{\left(1-x^{3(2 k)}\right)}{\left(1-x^{2 k}\right)} \frac{\left(1-x^{3.2(2 k-1)}\right)}{\left(1-x^{2(2 k-1)}\right)}
\end{aligned}
$$

this gives us two definitions, the first one is that the partition function $\frac{\theta_{4}\left(0, x^{3}\right)}{\theta_{4}(0, x)}$ is the generating function for partitions of $2 k$ prime to 3 in which all odd parts occur with even multiplicities. There is no restriction on the even parts. This follows from the first identity in which both even and odd parts are free of multiples of 3 . There is no restrictions on the other even parts. There is an other definition that follows from the second identity which gives the number of partitions of $2 k$ in which odd parts occur with multiplicity 2 or 4 . The even parts appear with at most twice. Using the second definition, then 6 can be written as

$$
6=4+2=4+1+1=2+2+1+1=3+3=2+1+1+1+1,
$$

and so $a(6)=6$. The above two definitions can be generalized for all $s$, this is given by following proposition

Proposition 5.1 The generating function $\frac{\theta_{4}\left(0, x^{s}\right)}{\theta_{4}(0, x)}$, for $s$ odd is the generating function for partitions of $2 k$ into parts prime to $s$. All odd parts occur with even multiplicities, there is no restrictions on the even parts. In general for all $s$, it is a generating function for partitions of $2 k$ into parts in which all odd parts occur with multiplicities $2,4, \cdots, 2(s-1)$. The even parts occur with at most $s-1$ times.

Proof: the proof is the same as we $\operatorname{did}$ for $s=3$,

$$
\begin{aligned}
\frac{\theta_{4}\left(0, x^{s}\right)}{\theta_{4}(0, x)} & =\prod_{k=1}^{\infty} \frac{\left(1+x^{k}\right)}{\left(1-x^{k}\right)} \frac{\left(1-x^{s k}\right)}{\left(1+x^{s k}\right)} \\
& =\prod_{k=1}^{\infty} \frac{\left(1-x^{s k}\right)}{\left(1-x^{k}\right)} \frac{\left(1-x^{s(2 k-1)}\right)}{\left(1-x^{(2 k-1)}\right)} .
\end{aligned}
$$


Next, change $x$ to $x^{2}$, then,

$$
\begin{aligned}
\frac{\theta_{4}\left(0, x^{2(s)}\right)}{\theta_{4}\left(0, x^{2}\right)} & =\prod_{k=1}^{\infty} \frac{\left(1-x^{2(s k)}\right)}{\left(1-x^{2 k}\right)} \frac{\left(1-x^{2 . s(2 k-1)}\right)}{\left(1-x^{2(2 k-1)}\right)} \\
& =\prod_{k=1}^{\infty} \frac{\left(1-x^{s(2 k)}\right)}{\left(1-x^{2 k}\right)} \frac{\left(1-x^{s .2(2 k-1)}\right)}{\left(1-x^{2(2 k-1)}\right)}
\end{aligned}
$$

so, for $s$ odd both the unrestricted even parts $1 /\left(1-x^{2 k}\right)$ and the odd parts that occur with even multiplicity $1 /\left(1-x^{2(2 k-1)}\right)$ are prime to $s$. From the last identity, we learn that for $s$ even or odd, the even part occur with at most $s-1$ while the odd parts occur with multiplicities $2,4, \cdots, 2(s-1)$.

A sequence A080054 [10, whose generating function is

$$
\frac{\theta_{4}\left(0, x^{2}\right)}{\theta_{4}(0, x)}=\prod_{k=1}^{\infty} \frac{\left(1+x^{2 k-1}\right)}{\left(1-x^{2 k-1}\right)}=\prod_{k=1}^{\infty}\left(1+x^{2 k-1}\right)\left(1+x^{k}\right)
$$

corresponds the number of partitions of $2 k$ in which the odd parts occur with multiplicity 2 . The even parts occur with multiplicity one. This definition was given by the author some time ago, to see it change $x$ to $x^{2}$ in the expression of the last product of equation (51). Setting $a=-1$ in the Lebesgue's identity 8 , corollary (2.7), then

$$
\frac{\theta_{4}\left(0, x^{2}\right)}{\theta_{4}(0, x)}=1+\sum_{k=1}^{\infty} \frac{2(1+x)\left(1+x^{2}\right) \cdots\left(1+x^{k-1}\right)}{(1-x)\left(1-x^{2}\right) \cdots\left(1-x^{k}\right)} x^{k(k+1) / 2} \text {. }
$$

It is interesting to note that this partition function together with the partition function of a parafermion of order 4 , which may be written as, $Z_{4}(\beta)=\prod_{k=1}^{\infty} \frac{\left(1+x^{2 k}\right)}{\left(1-x^{2 k-1}\right)}$ are nothing but the Ramond characters of the superconformal models $S M(2,8)$ [13], and in general the partition function $\frac{\theta_{4}\left(0, x^{k}\right)}{\theta_{4}(0, x)}$ for all $k$ is identified with the Ramond character $\hat{\chi}_{1,2 k}^{(2,4 k)}(q)$ of $S M(2,4 k)$. While writing this paper, we realized what there is a connection between our work and that of overpartitions used by Corteel and Lovejoy in combinatorial proofs of many q-series identities [11. There is also an alternative definition that arises from conformal field theory called jagged partitions [12]. An overpartition of $k$ is an ordered sequence of nonincreasing integers whose sum is $k$, where the first occurrence of each distinct integer (equivalently, the final occurrence) is overlined. Therefore, distinct parts are overlined and the unrestricted parts are non-overlined. For example the 8 overpatitions of 3 are

$$
3, \overline{3}, 2+1, \overline{2}+1,2+\overline{1}, \overline{2}+\overline{1}, \overline{1}+1+1,1+1+1 .
$$

This is exactly the number of partitions of 6 that we considered above. Note that this definition coincides with the tensor product of the bosonic and fermionic Fock spaces discussed above. If we denote $\bar{p}(k)$ the number of overpartitions, then

$$
\begin{aligned}
\frac{Z_{s}(\beta)}{\Delta_{s}^{+}(\beta)} & =\prod_{k=1}^{\infty} \frac{\left(1+x^{k}\right)}{\left(1-x^{k}\right)} \\
& =\sum_{k=0}^{\infty} \bar{p}(k) x^{k} \\
& =1+2 x+4 x^{2}+8 x^{3}+14 x^{4}+24 x^{5}+\cdots .
\end{aligned}
$$


The other interesting example, is when $s=3$ for which the number of partitions of 6 prime to 3 is 6 . In the language of overpartitions, we are dealing with overpartitions in which the parts are not divisible by 3 . Therefore, discarding 3 and $\overline{3}$ then the number of overpartitions of 6 is 6 . This also coincides with our second definition of $\frac{\theta_{4}\left(0, x^{3}\right)}{\theta_{4}(0, x)}$. The correspondence between overpartitions in which the parts are not divisible by $s$, and our definitions for partitions of $2 k$ prime to $s$ is as follows; First, if $s$ is odd, the number of overpartitions not divisible by $s$ is the number of partitions of $2 k$ prime to $s$. All odd parts occur with even multiplicities, there is no restrictions on the even parts. Second, if $s$ is even, then, the number of overpartitions not divisible by $s$ is the number of partitions of $2 k$ in which all odd parts occur with multiplicities $2,4, \cdots, 2(s-1)$. The even parts appear with at most $s-1$ times. In particular, if $s=2$, one has

$$
\begin{aligned}
\frac{\theta_{4}\left(0, x^{2}\right)}{\theta_{4}(0, x)} & =\prod_{k=1}^{\infty} \frac{\left(1+x^{k}\right)}{\left(1-x^{k}\right)} \frac{\left(1-x^{2 k}\right)}{\left(1+x^{2 k}\right)} \\
& =1+2 x+2 x^{2}+4 x^{3}+6 x^{4}+8 x^{5}+\cdots,
\end{aligned}
$$

this tells us that the number of overpartitions of 3 in which no part is divisible by 2 is 4 . we can see this by going back to the above example, then discarding partitions that involve 2 and $\overline{2}$. Using Andrews's multiple series transformation, and the Jacobi triple product [8], we can express $\frac{\theta_{4}\left(0, x^{s}\right)}{\theta_{4}(0, x)}$ as a multiple sum [11],

$$
\frac{\theta_{4}\left(0, x^{s}\right)}{\theta_{4}(0, x)}=\sum_{n_{s-1} \geq \cdots \geq n_{1} \geq 0} \frac{\prod_{j=0}^{n_{s-1}-1}\left(1+x^{j}\right) x^{1 / 2 n_{s-1}\left(n_{s-1}+1\right)+n_{s-2}^{2}+\cdots n_{1}^{2}}}{\prod_{j=0}^{n_{s-1}-n_{s-2}-1}\left(1-x^{j+1}\right) \cdots \prod_{j=0}^{n_{2}-n_{1}-1}\left(1-x^{j+1}\right) \prod_{j=0}^{n_{1}-1}\left(1-x^{j+1}\right)}
$$

The case $s=2$ follows from the Lebesgue's identity [8]. Because of the connection with the overpartitions, we conclude that our system whose partition function is $\frac{Z_{s}(\beta)}{\Delta_{s}^{ \pm}(\beta)}$, is given by the single sum through the Lebesgue's identity for $s$ even. However, for $s$ odd, the partition function is given by the multiple sum. We finish this section by noting that if the order of parafermions is even, then the system with the partition function (s-IGPPF) may be described in terms of overpartitions. This is clear from the expression of the partition function $\frac{1}{\Delta_{s}^{+}(\beta)}$ computed in the last section, see equation (37), then

$$
\begin{aligned}
Z_{F}(\beta) \Delta_{B}(s \beta) & =\prod_{k=1}^{\infty} \frac{\left(1+x^{k}\right)}{\left(1-x^{s k}\right)} \\
& =\prod_{k=1, k \nmid s / 2}^{\infty}\left(1+x^{k}\right) \prod_{k=1}^{\infty} \frac{1}{\left(1-x^{(s / 2) k}\right)},
\end{aligned}
$$

this partition function corresponds to a tensor (convolution) product of a certain bosonic Fock space with a fermionic Fock space, and has a similar form of overpartitions but with restrictions. From the above identity we have two definitions associated to this type of overpartitions. The first definition is like the above definition of overpartition of $k$ for the overlined parts. However, the non-overlined parts occur with multiplicity $s$. The second definition, implies that distinct parts which are overlined are not divisible by $s / 2$, and the non-overlined (unrestricted) parts are multiples of $s / 2$. If $s=4$, we know that the number of partition of 7 is 7 , therefore in terms of overpartitions, the 7 overpatitions of 7 are given by

$$
\overline{7}, \overline{6}+\overline{1}, \overline{5}+\overline{2}, \overline{4}+\overline{3}, 4+\overline{3}, \overline{4}+\overline{2}+\overline{1}, 4+\overline{2}+\overline{1} .
$$


or

$$
\overline{7}, 6+\overline{1}, \overline{5}+2,4+\overline{3}, 4+2+\overline{1}, \overline{3}+2+2,2+2+2+\overline{1},
$$

using the second definition.

\section{CONNECTION WITH THE RIEMANN GAS}

The Riemann gas is a system whose partition function is given by the Riemann zeta function [1],

$$
\begin{aligned}
\zeta(t) & =\frac{1}{\prod_{p}\left(1-p^{-t}\right)} \\
& =\sum_{n=1}^{\infty} 1 / n^{t},
\end{aligned}
$$

where the product is over all prime numbers $p$ and $t=\beta \omega$. The different partition functions obtained so far are related to the generating functions of partitions of integers. Therefore we may call these theories additive quantum theories to differentiate them from multiplicative quantum theories whose partition functions are related to the zeta function. Using the fact that bosonic partition functions for the Riemann gas and the Euler gas are given by infinite products. Therefore we may go from additive quantum theory to multiplicative quantum theory, simply by changing product over integers into product over primes, and changing the $x^{k}$ by $p^{-t}$. For example the fermionic partition function in the additive quantum theory is $Z_{F}(\beta)=\prod_{k=1}^{\infty}\left(1+x^{k}\right)$ which becomes in the multiplicative theory as

$$
\begin{aligned}
Z_{F}(t) & =\prod_{p}\left(1+p^{-t}\right) \\
& =\frac{\prod_{p}\left(1-p^{-2 t}\right)}{\prod_{p}\left(1-p^{-t}\right)} \\
& =\frac{\zeta(t)}{\zeta(2 t)} \\
& =\sum_{n=1}^{\infty} q(n) / n^{t},
\end{aligned}
$$

where $q(n)=1$, if $n$ is a square free, and $q(n)=0$ if $n$ has a squared factor. Therefore, in the Fock space of states, if we label the fermionic states $\prod_{i}\left(f_{i}^{\dagger}\right)^{\beta_{i}}|\tilde{\emptyset}\rangle$ by integers, that is, $|n\rangle, n=\prod_{i} p_{i}^{\beta_{i}}$ then $\beta_{i}$ must take the values 0 or 1 . Note that $q(n)$ is related to the Möbius function $\mu(n)$, by $q(n)=|\mu(n)|[24]$ where $\mu(1)=1, \mu(n)=(-1)^{k}$ if $n$ is the product of different primes, and $\mu(n)=0$, if $\beta_{i}>1$. Similarly, for parafermions, using equation (16), the parafermionic partition 
function for the Riemann gas can be written as,

$$
\begin{aligned}
Z_{s}(t) & =\prod_{p}\left(1+p^{-t}+p^{-2 t}+\cdots+p^{-(s-1) t}\right) \\
& =\frac{\prod_{p}\left(1-p^{-s t}\right)}{\prod_{p}\left(1-p^{-t}\right)} \\
& =\frac{\zeta(t)}{\zeta(s t)} \\
& =\sum_{n=1}^{\infty} q_{s}(n) / n^{t}
\end{aligned}
$$

where $q_{s}(n)$ takes the values 0 or 1 , depending if $n$ has an sth power as a factor or not. All of these partitions functions are well known for the multiplicative quantum theory [4]. Next, we will obtain the partition functions that are analogous to those in the additive quantum theory. From equation (25), the graded partition function for $s$ even is

$$
\begin{aligned}
\Delta_{s}^{+}(t) & =\prod_{p}\left(1-p^{-t}+p^{-2 t}-\cdots-p^{-(s-1) t}\right) \\
& =\frac{\prod_{p}\left(1-p^{-s t}\right)}{\prod_{p}\left(1+p^{-t}\right)} \\
& =\frac{\zeta(2 t)}{\zeta(t) \zeta(s t)} \\
& =\sum_{n=1}^{\infty} \mu_{s}(n) / n^{t}
\end{aligned}
$$

where we have introduced the truncated Möbius function $\mu_{s}(n)$ function to differentiate it from the ordinary Möbius function $\mu(n)$. The truncated Möbius function $\mu_{s}(n)$ would be zero if $n$ is divisible by the sth power of some prime, so we may call it the Möbius function of order $s$. For example if $s=2$, we obtain;

$$
\frac{1}{\zeta(t)}=\sum_{n=1}^{\infty} \mu(n) / n^{t},
$$

that is, the usual expression for the inverted zeta function. For $s$ odd, then, the graded partition function is

$$
\begin{aligned}
\Delta_{s}^{-}(t) & =\prod_{p}\left(1-p^{-t}+p^{-2 t}-\cdots+p^{-(s-1) t}\right) \\
& =\frac{\prod_{p}\left(1+p^{-s t}\right)}{\prod_{p}\left(1+p^{-t}\right)} \\
& =\frac{\zeta(2 t) \zeta(s t)}{\zeta(t) \zeta(2 s t)} \\
& =\sum_{n=1}^{\infty} \mu_{s}(n) / n^{t}
\end{aligned}
$$


From these two equations, the counter parts of the inverted graded partition functions (28), (29) follows. Thus, if $s$ is even, the inverted parafermionic partition function may be written as

$$
\begin{aligned}
\frac{1}{\Delta_{s}^{+}(t)} & =Z_{B}(s t) Z_{F}(t) \\
& =\frac{\prod_{p}\left(1+p^{-t}\right)}{\prod_{p}\left(1-p^{-s t}\right)} \\
& =\frac{\zeta(t) \zeta(s t)}{\zeta(2 t)} \\
& =\prod_{p}\left(\sum_{j=0}^{\infty} c(j)^{+} p^{-t j}\right) .
\end{aligned}
$$

Where $c(0)^{+}=1, c(j)^{+}=1$ if $j$ is congruent to 0,1 modulo $s$ and $c(j)^{+}=0$ if $j$ is congruent to $2,3, \cdots,(s-1)$ modulo $s$. Therefore, if we write the partition function $\frac{\zeta(t) \zeta(s t)}{\zeta(2 t)}$ additively as the Dirichlet series $\sum_{n=1}^{\infty} a(n)^{+} n^{-t}$, then the coefficients $a_{n}^{+}$are multiplicative with $a^{+}\left(p_{1}^{j_{1}} \cdots p_{r}^{j_{r}}\right)=$ $c\left(j_{1}\right)^{+} \cdots c\left(j_{r}\right)^{+}$. In particular $a(n)^{+}=0$ if $n$ is divisible by some prime to an exponent which is congruent to $2,3, \cdots,(s-1)$ modulo $s$, and is equal to 1 otherwise. As a consequence, the Dirichlet formula for any $s$, may be written as,

$$
\frac{\zeta(t) \zeta(s t)}{\zeta(2 t)}=\sum_{n=1}^{\infty} \frac{1}{n^{t}}
$$

where the exponents in $n=\prod_{i} p_{i}^{r_{i}}, r_{i}$, are congruent to 0,1 modulo $s$. In terms of the quantum states $|n\rangle$, in the Fock space labeled by an integer $n, n=\prod_{i} p_{i}^{r_{i}}$, the states for which the exponents $r_{i}$ are congruent to $2,3, \cdots,(s-1)$ modulo $s$, are missing. The only states present in the Fock space are those for which the exponents are congruent to 0,1 modulo $s$. If the the order of parafermions $s$ is odd, then the inverted parafermionic partition function for the Riemann gas would be;

$$
\begin{aligned}
\frac{1}{\Delta_{s}^{-}(t)} & =Z_{F}(t) \Delta_{B}(s t) \\
& =\frac{\prod_{p}\left(1+p^{-t}\right)}{\prod_{p}\left(1+p^{-s t}\right)} \\
& =\frac{\zeta(t) \zeta(2 s t)}{\zeta(2 t) \zeta(s t)} \\
& =\prod_{p}\left(\sum_{j=0}^{\infty} c(j)^{-} p^{-t j}\right)
\end{aligned}
$$

where $c(0)^{-}=1, c(j)^{-}=1$ if $j$ is congruent to 0,1 modulo $2 s, c(j)^{-}=-1$ if $j$ is congruent to 0,1 modulo $s$. Alternatively we may write, $c(j)^{-}=(-1)^{[j / s]}$, where $[x]$ denotes the integer part of $x$, and $c(j)^{-}=0$ if $j$ is congruent to $2,3, \cdots,(s-1)$ modulo $s$. In this case the Dirichlet series associated with the partition function $\frac{\zeta(t) \zeta(2 s t)}{\zeta(2 t) \zeta(s t)}$, is $\sum_{n=1}^{\infty} a_{n}^{-} n^{-t}$, where the coefficients $a_{n}^{-}=0$, if $n$ is divisible by some prime to an exponent which is congruent to $2,3, \cdots,(s-1)$ modulo $s$. It is equal to \pm 1 otherwise. Note that the minus one is due to the graded bosonic partition function $\Delta_{B}(s t)$. Therefore, the Dirichlet series takes the following expression;

$$
\frac{\zeta(t) \zeta(2 s t)}{\zeta(2 t) \zeta(s t)}=\sum_{n=1}^{\infty} \frac{(-1)^{\left[r_{1} / s\right]+\left[r_{2} / s\right]+\cdots+\left[r_{k} / s\right]}}{n^{t}}
$$


where the exponents $r_{i}$ in $n=\prod_{i} p_{i}^{r_{i}}$, are not congruent to $2,3, \cdots,(s-1)$ modulo $s$. In this case, the Fock space of states labeled by an integer $n, n=\prod_{i} p_{i}^{r_{i}}$, in which the exponents $r_{i}$ are congruent to $2,3, \cdots,(s-1)$ modulo $s$ are missing, otherwise, the states would be present. We give finally, the analogue of the generating functions $\frac{1}{\theta_{4}(0, x)}$ and $\frac{\theta_{4}\left(0, x^{s}\right)}{\theta_{4}(0, x)}$, given by equations (35), (36) respectively, for the Riemann gas. Following the same procedure as above, the analogue of $\frac{1}{\theta_{4}(0, x)}$ is;

$$
\begin{aligned}
\frac{Z_{s}(t)}{\Delta_{s}^{+}(t)} & =Z_{B}(t) Z_{F}(t), \\
& =\frac{\prod_{p}\left(1+p^{-t}\right)}{\prod_{p}\left(1-p^{-t}\right)} \\
& =\frac{\zeta(t)^{2}}{\zeta(2 t)} \\
& =\prod_{p}\left(1+2 p^{-t}+2 p^{-2 t}+2 p^{-3 t}+\cdots\right), \\
& =\prod_{p}\left(\sum_{j=0}^{\infty} 2^{\nu\left(p^{j}\right)} p^{-t j}\right), \\
& =\sum_{n=1}^{\infty} 2^{\nu(n)} / n^{t},
\end{aligned}
$$

where the sum is over all integers $n$, and $\nu(n)$ is the number of distinct prime factors of $n$, and so $\nu(1)=0$ and if $n=\prod_{i=1}^{k} p_{i}^{r_{i}}$, then $\nu(n)=k$. This is exacly the partition function associated with the unitary twisting of two identical bosonic gases [3]. Here, we obtain this result by mixing a paraferminic system with a system whose partition function is the inverted graded partition function. As a result, the analogue of $\frac{1}{\theta_{4}(0, x)}$ in the Riemann gas is $\frac{\zeta(t)^{2}}{\zeta(2 t)}$. We may derive equation (66), by computing the total partition function $Z_{T}(\beta)=\operatorname{Tr} \exp -\beta H$, where $H$ is the total Hamiltonian, i.e, $H=H_{B}+H_{F}$, and the trace is taken over special kind of normalized states that we denote by $|n, d\rangle$, here, $n$ is the total number eigenvalue such that the numbers $d$ are divisors of $n$, and square free [2]. The state $|n, d\rangle$, in the multiplicative quantum theory has an energy $E_{n}=\beta \log (n)$. Writing $E_{n}=\langle n, d|H| n, d\rangle$, and using the notation $\sum_{d \mid n}^{\prime}$ to mean that the sum is over all divisors $d$ of $n$ which are square free, then the total partition function becomes,

$$
\begin{aligned}
Z_{T}(\beta) & =\sum_{n=1}^{\infty}\left(\sum_{d \mid n}^{\prime}\langle n, d|\exp -\beta H| n, d\rangle\right) \\
& =\sum_{n=1}^{\infty} \exp -\beta E_{n}\left(\sum_{d \mid n}^{\prime}\langle n, d \mid n, d\rangle\right), \\
& =\sum_{n=1}^{\infty} \exp -(\beta \omega \log (n)) \sum_{d \mid n} q(d),
\end{aligned}
$$

where $q(d)=\langle n, d \mid n, d\rangle$ is equal to 1 , when $d$ is a square free, and is equal to 0 , when $d$ has squared factor. Note that if we did not have the constraint that $d$ is a square free but just a divisor of $n$, then

$$
\sum_{d \mid n}\langle n, d \mid n, d\rangle=\sum_{d \mid n} 1=\tau(n)
$$


that is, the number of divisors of $n$. However, in our case the sum is given by the number of unitary divisors of $n$ through the identity $\sum_{d \mid n} q(d)=2^{\nu(n)}$, recall that $d$ is a unitary divisor of $n$ if the greatest common divisor of $d$ and $\frac{n}{d}$ is one. Using the latter identity, and setting $t=\beta \omega$ in the above equation (67), gives

$$
Z_{T}(\beta)=\sum_{n=1}^{\infty} 2^{\nu(n)} / n^{t} .
$$

This is exactly equation (66), this would be, the the analogue of the overpartition generating function in the additive number theory. As an example, the bosonic and Fermionic states in the Fock space associated to the mixed system, whose partition function is given by equation (66), up $n=20$ are,

$$
|1\rangle|,| 2\rangle,|3\rangle,|4\rangle,|5\rangle, \cdots,|20\rangle
$$

for the bosonic states, and

$$
|1\rangle|,| 2\rangle,|3\rangle,|5\rangle,|6\rangle, \cdots,|19\rangle,
$$

for the fermionic states, note that in this case, the integers are square free. One can see that the tensor product of these states defined by multiplying the bosonic labels times the fermionic labels in this order, such that the total number $n=20$, would corresponds to the following states,

$$
|20.1\rangle,|10.2\rangle,|4.5\rangle,|2.10\rangle \text {. }
$$

Using the notation $|n, d\rangle$, where $d$ is a square free divisor of 20 , then, the 4 states are;

$$
|20,10\rangle,|20,5\rangle,|20,2\rangle,|20,1\rangle
$$

alternatively in terms of unitary divisors $d^{*}(20)$, this is written as,

$$
|20,20\rangle,|20,5\rangle,|20,4\rangle,|20,1\rangle
$$

this simply says, that the number of square free divisors of $n$ is the same as the number of unitary divisors $d^{*}(n)$ of $n$. Therefore, we may alternatively denote the states $|n, d\rangle$, by $\left|n, d^{*}(n)\right\rangle$. Alternatively, we may relate the additive and multiplicative theories through the correspondence between the additive generating theta function $\theta(t)$ and the multiplicative Riemann zeta function $\zeta(t)$. This correspondence states that the the Riemann zeta function is the Mellin transform of $(\theta(t)-1)$ [25. The Mellin transform of a given function $f(t)$ on the positive real line is defined by the formula $\Phi(s):=\int_{0}^{\infty} f(t) t^{s-1} d t$ for values of $s$ such that the integral converges. We will show up to some factors that the Mellin transform of the theta Jacobi $\theta_{4}(t)$ is the alternating Riemann zeta function, also called the Dirichlet Eta function $\eta(s)=\sum_{n=1}^{\infty}(-1)^{n+1} \frac{1}{n^{s}}$. This result was given in [26], however, our derivation is explicit and closely related to the derivation that makes the connection between $\theta(t)$ and $\zeta(t)$ functions. To this end, let us first write $\theta_{4}(t)=$ $\sum_{n=-\infty}^{\infty}(-1)^{n} e^{-n^{2} \pi t}$ in terms of $\theta(t)=1+2 \sum_{n=0}^{\infty} e^{-n^{2} \pi t}$. The theta function $\theta_{4}(t)$ may be written explicitly in the form

$$
\begin{aligned}
\theta_{4}(t) & =1+2\left(\sum_{n \text { even }}^{\infty} e^{-n^{2} \pi t}-\sum_{n \text { odd }}^{\infty} e^{-n^{2} \pi t}\right) \\
& =1+2\left(2 \sum_{n=1}^{\infty} e^{-4 n^{2} \pi t}-\sum_{n=1}^{\infty} e^{-n^{2} \pi t}\right) \\
& =2 \theta(4 t)-\theta(t)
\end{aligned}
$$


To find the Mellin transform of $\theta_{4}(t)$, we use the fact that the theta function $\theta(t)$ satisfy the following functional equation

$$
\theta(t)=t^{-1 / 2} \theta(1 / t)
$$

and so,

$$
\theta(4 t)=\frac{1}{2} t^{-1 / 2} \theta(1 / 4 t)
$$

Thus, near $t=0, \theta(t)$ looks like like $t^{-1 / 2}$, and $\theta(4 t)$, looks like $\frac{1}{2} t^{-1 / 2}$, while for large $t$ both $\theta(t)$ and $\theta(4 t)$ are asymptotic to 1 . Usually In obtaining the zeta function $\zeta(t)$ from the theta function, we replace $s$ by $s / 2$ in the definition of the Miller transform. Therefore,the Mellin transform may be written as

$$
\left.\left.\Phi(s)(\theta(4 t)):=\int_{1}^{\infty} t^{s / 2-1}(\theta(4 t)-1) d t\right)+\int_{0}^{1} t^{s / 2-1}\left(\theta(4 t)-\frac{1}{2} t^{-1 / 2}\right) d t\right) .
$$

Note that the first integral is convergent for large $t$, while the second integral is convergent near $t=0$. Now, it is safe to integrate term by term in the above integrals and at the end we sum over $n$ to obtain

$$
\begin{aligned}
\Phi(s)(\theta(4 t)): & \left.\left.=\int_{1}^{\infty} t^{s / 2-1}(\theta(4 t)-1) d t\right)+\int_{0}^{1} t^{s / 2-1}\left(\theta(4 t)-\frac{1}{2} t^{-1 / 2}\right) d t\right) \\
& =\frac{2}{\pi^{s / 2} 2^{s}} \Gamma(s / 2) \zeta(s)+\frac{2}{s}+\frac{1}{1-s}
\end{aligned}
$$

Now, the Mellin transform of $\theta(t)$ is given by the formula

$$
\begin{aligned}
\Phi(s)(\theta(t)): & \left.\left.=\int_{1}^{\infty} t^{s / 2-1}(\theta(t)-1) d t\right)+\int_{0}^{1} t^{s / 2-1}\left(\theta(t)-t^{-1 / 2}\right) d t\right) \\
& =\frac{2}{\pi^{s / 2}} \Gamma(s / 2) \zeta(s)+\frac{2}{s}+\frac{2}{1-s} .
\end{aligned}
$$

This is the correspondence between the additive theta function and the multiplicative Riemann zeta function. Finally, the Mellin transform of the theta function $\theta_{4}(t)$ may be given by the following formula

$$
\begin{aligned}
\Phi(s)\left(\theta_{4}(t)\right) & =2 \Phi(s)(\theta(4 t))-\Phi(s)(\theta(t)) \\
& \left.=\frac{2}{\pi^{s / 2}} \Gamma(s / 2)\left(2^{1-s}-1\right) \zeta(s)\right)+\frac{2}{s} \\
& =-\frac{2}{\pi^{s / 2}} \Gamma(s / 2) \eta(s)+\frac{2}{s} .
\end{aligned}
$$

Therfore, the altrnating theta function $\theta_{4}(0, x)$ is related to the alternating Riemann zeta function $\eta(s)$. Because of the Dirichlet series $\sum_{n=1}^{\infty} 2^{\nu(n)} / n^{t}$ representation of $\frac{\zeta(t)^{2}}{\zeta(2 t)}$, then, one would follow formally, similar steps as in [27] page 167. Given the Drichlet series $\Psi_{f}(s)=\sum_{n=1}^{\infty} \frac{c(n)}{n^{s}}$, where $f(z)=\sum_{n=0}^{\infty} e^{2 \pi i z}$ is a modular form of weight $2 k, k>0$, Hecke proved that the series $\Psi_{f}(s)$ can be analytically extended to a meromorphic function in the whole complex pane, and the function defined by $\Lambda_{f}(s)=(2 \pi)^{-s} \Gamma(s) \Psi_{f}(s)$ satisfies :

$$
\Lambda_{f}(s)=(-1)^{k} \Lambda_{f}(2 k-s) .
$$


F. Diamonde informed me that from the Euler product of the Dirichlet series, see equation (66), the Euler factors are not the sort that could arise from a modular form. therefore, one should look for a non direct method, and can not be done as in the correspondence between the Riemann zeta function and the theta function.

Finally, we would like to find the analogue of the partition function $\frac{\theta_{4}\left(0, x^{s}\right)}{\theta_{4}(0, x)}$, first the theta for the Riemann gas, may be written for $s$ odd, as follows;

$$
\begin{aligned}
\frac{Z_{s}(t)}{\Delta_{s}^{-}(t)} & =Z_{B}(t) Z_{F}(t) \Delta_{B}(s t) \Delta_{F}(s t) \\
& =\frac{\prod_{p}\left(1+p^{-t}\right)}{\prod_{p}\left(1-p^{-t}\right)} \frac{\prod_{p}\left(1-p^{-s t}\right)}{\prod_{p}\left(1+p^{-s t}\right)} \\
& =\frac{\zeta(t)^{2}}{\zeta(2 t)} \frac{\zeta(2 s t)}{\zeta(s t)^{2}}, \\
& =\prod_{p} \frac{\left(1+2 p^{-t}+2 p^{-2 t}+2 p^{-3 t}+\cdots+2 p^{-(s-1) t}+p^{-s t}\right)}{\left(1+p^{-s t}\right)} \\
& =\prod_{p}\left(\sum_{j=0}^{\infty} c(j) p^{-t j}\right),
\end{aligned}
$$

where $c(0)=1, c(j)=0$ if $j>0$ and $j$ is divisible by $s$, and $c(j)=2(-1)^{[j / s]}$ if $j$ is not divisible by $s$, where $[x]$ denotes the integer part of $x$. Therefore, if we write the partition function $\frac{\zeta(t)^{2}}{\zeta(2 t)} \frac{\zeta(2 s t)}{\zeta(s t)^{2}}$, additively as the Dirichlet series $\sum_{n=1}^{\infty} a(n) n^{-t}$. Then the multiplicative function $a(n)$ takes the following values, $a(n)=0$ if $n$ is divisible by some prime to an exponent which is a multiple $s$. Otherwise, it is equal to \pm 1 times power of 2 . Therefore, the quantum states of the Riemann gas for $s$ odd, labeled by an integer $n$, where $n=\prod_{i} p_{i}^{r_{i}}$ are free of primes whose powers are divisible by $s$. This is similar to the Euler gas whose partition function is $\frac{\theta_{4}\left(0, x^{s}\right)}{\theta_{4}(0, x)}$, with $s$ odd. Recall, that in this case, the subscripts of the bosonic and fermionic creation operators are prime to $s$. By doing explicit computation for different values of $s$, it turns out, that a general formula for $\frac{\zeta(t)^{2}}{\zeta(2 t)} \frac{\zeta(2 s t)}{\zeta(s t)^{2}}$ may be written as;

$$
\begin{aligned}
\frac{\zeta(t)^{2}}{\zeta(2 t)} \frac{\zeta(2 s t)}{\zeta(s t)^{2}} & =\prod_{p} \frac{\left(1+2 p^{-t}+2 p^{-2 t}+2 p^{-3 t}+\cdots+2 p^{-(s-1) t}+p^{-s t}\right)}{\left(1+p^{-s t}\right)} \\
& =\sum_{n=1}^{\infty} \frac{(-1)^{\left[k_{1} / s\right]+\left[k_{2} / s\right]+\cdots+\left[k_{r} / s\right]} 2^{\nu(n)}}{n^{t}}
\end{aligned}
$$

where the sum is taken over all $n=p_{1}^{k_{1}} \cdots p_{r}^{k_{r}}$, such that the exponents $k_{i}$ 's are not divisible by $s$.

\section{DISCUSSION}

In this paper, we derived the Euler theorem as well as a theorem in the theory of partitions which may be stated as follows; the number of partitions of $k$ in which no parts appear more than $s-1$ times equals the number of partitions of a positive integer $k$ such that no parts is divisible by $s$. The derivation of the Euler theorem and its generalization is based on the fact that the 
bosonic Hamiltonian of a non-interacting quantum field theory may be decomposed into bosonic Hamiltonian whose frequencies are twice ( $s$-times) as much as the original Hamiltonian, and a fermionic (prafermionic) Hamiltonian. By realizing that the graded fermionic partition function $\Delta_{F}(\beta)=\prod_{k=1}^{\infty}\left(1-x^{k}\right)$ is obtained from fermionic partition function by simply changing $x^{k}$ to $-x^{k}$. Then the expressions for the graded parafermionic partition functions were obtained whose inverse would correspond to to bosonic(fermionic) partition function depending on the order of parafermions being even or odd, respectively. Both of these partition functions, generate partition of numbers with given restrictions. The generating functions that we have obtained are general in the sense that when the order of the parafermions is two, then our generating function coincides with the Euler generating function. These generating functions give rise to some sequences of partitions [10]. If the order of the parafermions is infinite, then the inverse of the parafermionic partition functions $\left(s\right.$-IGPPF) $\frac{1}{\Delta_{s}^{ \pm}(\beta)}$ tend to a fermionic partition function. Therefore, as the boson is an infinite order parafermion, a fermion would be an infinite order inverted graded parafermion. The Euler theorem equates the number of distinct partitions with the number of unrestricted odd partitions. The proof of this theorem is simple in number theory, this is also the case through the factorization of the fermionic partition function into a bosonic partition function and a graded fermionic partition function. One may look at it differently, and write $Z_{f}(\beta)=\Delta_{F}(2 \beta) / \Delta_{F}(\beta)$ from which the graded fermionic partition function is $\Delta_{F}(2 \beta)=Z_{f}(\beta) \Delta_{F}(\beta)$, so mixing the fermionic system with the graded fermionic system at thermal equilibrum at a given temperature $\beta$ is the same as a graded parafermionic system whose temperature is doubled. This also happens in the case of quantum field theory with a logarithmic spectrum [4, in which the term duality was used to characterize the identities among arithmetic quantum theories. The above mixing is a special case of our relation $\frac{1}{\Delta_{s}^{+}(\beta)}=Z_{B}(s \beta) Z_{F}(\beta)$. Also, we may write the fermionic partition function in terms of parafermionic partition functions as $Z_{f}(\beta)=\Delta_{F}(s \beta) / \Delta_{s}^{+}(\beta)$ or equivalently $Z_{f}(\beta)=\frac{1}{\Delta_{B}(s \beta) \Delta_{s}^{-}(\beta)}$. Mixing a parafermion system of even order $s$ with a system whose partition function is $\frac{1}{\Delta_{s}^{+}(\beta)}$, was shown to be equivalent to mixing fermions and bosons. For $s$ odd, the system obtained is still a mixing of fermions and bosons but with some states missing in the Fock space of states. Also, in this case we related our partitions functions with partition theory. We have shown that these partition functions are written in terms of the Jacobi theta function $\theta_{4}(0, x)$, in particular, if $s$ is even, the partition function is $1 / \theta_{4}(0, x)$, while if $s$ is odd then the partition may be written as $\theta_{4}\left(0, x^{s}\right) / \theta_{4}(0, x)$. Here, it is interesting to note that the sequences generated by the partition functions (4-IGPPF), and $1 / \theta_{4}(0, x)$ appear in the counting of the Moore-Read edge spectra [14]. On the other hand, the sequences associated with the partition function of a parafermion of order $4, Z_{4}(\beta)$ with partition function $\theta_{4}\left(0, x^{2}\right) / \theta_{4}(0, x)$ are those that appear in the Ramond characters of the superconformal models $S M(2,8)$ [13]. In general the partition function $\frac{\theta_{4}\left(0, x^{s}\right)}{\theta_{4}(0, x)}$ for all $s$ is identified with the Ramond character $\hat{\chi}_{1,2 s}^{2,4 s)}(x)$ of $S M(2,4 s)$. We have also given a general definition of the partition function $\frac{\theta_{4}\left(0, x^{s}\right)}{\theta_{4}(0, x)}$ for all $s$ in terms of partition theory. A connection with overpartitions [11] in which the parts are not divisible by $s$ was also given. Using the expression for the parafermionic partition function given by Eq. (13), one has $\Delta_{F}(s \beta)=Z_{s}(\beta) \Delta_{F}(\beta)$ for any $s$, this identity will be a trivial identity when $s$ goes to infinity as both bosonic and graded fermionic partition functions cancel each other, this is equivalent to the Witten index which is a complete cancelation between boson and fermions. We have seen that the square of a bosonic operator is not bosonic operator but a parafermion with order parastatistics $p=1 / 2, p=3 / 2$. For a single oscillator, the norm of the states is positive in agreement with the expressions of the partition functions. For the quantum field theory associated with these oscillators, the norm is not always positive and so we expect to see this at the level of the partition function. In the case of a parastatistical systems obeying the Green's trilinear relations [7] with 
the total number of particles $N$, and with finite number of quantum states denoted by $M$. Then the grand canonical partition function turns out to be given in terms of the Schur's function [28],

$$
s_{\lambda}\left(x_{1}, x_{2}, \cdots, x_{M}\right)=\frac{\operatorname{det}\left(x_{i}^{\lambda_{j}+M-j}\right)}{\operatorname{det}\left(x_{i}^{M-j}\right)} ; 1 \leq i, j \leq M,
$$

where $\lambda=\left(\lambda_{1}, \lambda_{2}, \cdots, \lambda_{M}\right)$ is a partition of length $l(\lambda) \leq N$. Explicitly, the grand canonical partition function for parabose statistics of order $s$ is

$$
Z_{B}^{s}=\sum_{l(\lambda) \leq s} s_{\lambda}(x)
$$

where the sum is over all partitions $\lambda$ such that $l(\lambda) \leq s$. For parafermi statistics, the grand canonical partition function may be written as

$$
Z_{F}^{s}=\sum_{\lambda_{1} \leq s} s_{\lambda}(x)=\frac{\operatorname{det}\left(x_{i}^{s+2 M-j}-x_{i}^{j-1}\right)}{\operatorname{det}\left(x_{i}^{2 M-j}-x_{i}^{j-1}\right)}, 1 \leq i, j \leq M
$$

here, $\lambda_{1}=l(\lambda /)$ and $\lambda^{\prime}$ being the conjugate partition of the partition $\lambda$. If the order of the parastatistics is infinite then, there is no restrictions on the above sums and as a result [29], one obtains

$$
Z_{B}^{\infty}=Z_{F}^{\infty}=\prod_{i} \frac{1}{\left(1-x_{i}\right)} \prod_{i<j} \frac{1}{\left(1-x_{i} x_{j}\right)} .
$$

This equality is a expected from the Green's trilinear relations in which for $s \rightarrow \infty$, the parabose and the parafermi statistics are identical [30]. The parabosonic partition function $Z_{B}^{\infty}$ was derived earlier [31], using the standad states of Ohnuki and Kamefuchi [32], the above partition function was then used to obtain the expression for the partition function of the parabosonic string $Z$, as well as the large $n$ level density $d_{n}$ given by $Z=\sum d_{n} x^{n}$. The parafermi partition function of order $s=3$ with two level system was computed in [33. However, for $s>3, M>s$, the expressions for the partition functions are lacking.

The asymptotic partition of the parafermionc partition function

$$
Z_{s}(\beta)=\prod_{k=1}^{\infty}\left(\frac{1-x^{k s}}{1-x^{k}}\right)=\sum_{n=0}^{\infty} p(n, s) x^{n}
$$

was carried out by Hagis [34, using the Hardy-Ramanujan-Rademacher method. And for $n \gg s$, his results are,

$$
p(n, s) \sim \frac{\sqrt{1} 2 s^{1 / 4}}{(1+s)^{3 / 4}(24)^{3 / 4}} e^{\pi \sqrt{2 s /[3(1+s)]}} \sqrt{n} .
$$

For the parabosonic partition function discussed in this paper, that is,

$$
\prod_{k=1}^{\infty}\left(1+\frac{x^{k s}}{1-x^{k}}\right)=\sum_{n=0}^{\infty} p(n, s)^{\prime} x^{n}
$$

One would like to give the meaning to $p(n, s)^{\prime}$ in terms of partitions, as well as to consider the large $n$ limit for $p(n, s)^{\prime}$. One may follow the alternative derivation of Hagis formula, given in [35] in which they obtained an expression for $\ln p(n, s)$ by mapping a mathematical problem to a physical problem. 
In the last section we obtained the counter parts of the partitions functions associated with the Euler gas. This was possible, because both the Euler gas and the Riemann gas have a bosonic partition function that are infinite products one is over integers (the Euler partition function) and the other is over primes(the Riemann zeta function). In particular, the counter part of $\frac{1}{\theta_{4}(0, x)}$ and $\frac{\theta_{4}\left(0, x^{s}\right)}{\theta_{4}(0, x)}$, are $\frac{\zeta(t)^{2}}{\zeta(2 t)}, \frac{\zeta(t)^{2}}{\zeta(2 t)} \frac{\zeta(2 s t)}{\zeta(s t)^{2}}$, respectively. Thus, we see that the analogue of the Jacobi Theta function is $\frac{\zeta(2 t)}{\zeta(t)^{2}}$. In terms of the Fock space of states, for the Euler gas, the quantum states are constructed from the operators $\left(a_{k}^{\dagger}\right)$, such that $k$ are prime to $s$. For the Riemann gas, the quantum states $|n\rangle$ are those states for which the integers $n$ are free of primes whose powers are multiples of $s$. Also, note that all the the partition functions obtained for the the Riemann gas are written as a Dirichlet series which would be the analogue of the Lebesgue-Cauchy and the Andrews's multiple sums [11. We conclude this discussion, by giving other identities and make connection between the generating functions that come up in the additive and the multiplicative number theory. From equations (60) and (61), we have

$$
\sum_{n=1}^{\infty} \mu_{s}(n) / n^{t}=\frac{\zeta(2 t)}{\zeta(t) \zeta(s t)} s \text { even }
$$

and

$$
\sum_{n=1}^{\infty} \mu_{s}(n) / n^{t}=\frac{\zeta(2 t) \zeta(s t)}{\zeta(t) \zeta(2 s t)}, \quad s \text { odd }
$$

An explicit computation for $s$ odd in the first formula, and $s$ even in the second formula give the following identities,

$$
\begin{gathered}
\frac{\zeta(2 t)}{\zeta(t) \zeta(s t)}=\sum_{n=1}^{\infty} 2^{\nu_{s}(n)} \lambda(n) / n^{t}, \quad s \text { odd } \\
\frac{\zeta(2 t) \zeta(s t)}{\zeta(t) \zeta(2 s t)}=\sum_{n=1}^{\infty} 2^{\nu_{s}(n)} \lambda(n) / n^{t}, \quad s \text { even }
\end{gathered}
$$

where we have introduced the function $\nu_{s}(n)$, which is equal to $\nu(n)$, when the prime powers $r_{i} \geq s$, and if some $r_{i}<s$, then $\nu_{s}(n)=k-\#\left(\hat{k_{i}}\right)$, \#( $\left(\hat{k_{i}}\right)$ is the number of prime whose powers are less then $s$. When $s=1, \nu_{s}(n)=\nu(n)$ and we have the well known formula [5],

$$
\frac{\zeta(2 t)}{\zeta(t)^{2}}=\sum_{n=1}^{\infty} 2^{\nu(n)} \lambda(n) / n^{t}
$$

We saw that in the last section that the generating function

$$
\frac{\zeta(t)^{2}}{\zeta(2 t)}=\sum_{n=1}^{\infty} 2^{\nu(n)} / n^{t}
$$

is the counter part of the generating function $\prod_{k=1}^{\infty} \frac{\left(1+x^{k}\right)}{\left(1-x^{k}\right)}$. This is known to be given by the Cauchy formula, see equation (41), however, to make the correspondence closer for the sum given by equation (66), we rewrite the latter product as, $\prod_{k=1}^{\infty}\left(1+\frac{2 x^{k}}{\left(1-x^{k}\right)}\right)$. then an explicit computation shows that the general expression for this product can be written as

$$
\prod_{k=1}^{\infty} \frac{\left(1+x^{k}\right)}{\left(1-x^{k}\right)}=1+\sum_{n \geq 1} \sum_{i \leq n} \sum_{\substack{n_{1}>n_{2}>\ldots>n_{i}>0 \\ n_{1}+\ldots+n_{i}=n}} \frac{2^{i} x^{n}}{\left(1-x^{n_{1}}\right) \ldots\left(1-x^{n_{i}}\right)}
$$


Here, $n_{1}>n_{2}>\ldots>n_{i}$ are the part sizes of an overpartition, and $i$ be the number of part sizes (the number of distinct parts of $n$ ) and $n=n_{1}+\ldots+n_{i}$ be the sum of the part sizes. We have checked this formula for many values. It is interesting to note that from the above formula, and its counter part that the power of 2 , is the number of distinct parts for all partitions of $n$ in the additive number theory. In the multiplicative number theory, the power of 2 is the number of distinct primes factors of $n$. The above formula, shows clearly that the expansion coefficients, are always even for $n \geq 1$. Also, the above formula suggests that One may relate an overpatition $\tilde{p}(n)$ with the ordinary partition $p(n), 2^{i}$ counts the number of distinct overpartitions, and $i$ is the number of distinct parts in the partition of $n$, so if $p_{i}(n)$ is the number of distinct parts in the partition of $n$, then from our formula, one may write

$$
\tilde{p}(n)=\sum_{i} 2^{i} p_{i}(n)
$$

For example $p(4)=5,4=3+1=2+2=2+1+1=1+1+1+1$, while $p_{1}(n)=3, p_{2}(n)=2$, and so $\tilde{p}(4)=14$ in agreement with the overpartition of 4 . Slight modification of this formula may be used to count the number of overpations with restrictions. We know from the expression of overpartition modulo 2, that the the number of overpartions of 4 in which no parts is divisible by 2 is 6 . We can checke this using the above example by discarding the distinct parts divisible by 2 . If $\tilde{p}(n)^{2}, p_{i}^{2}(n)$ denote the number overpartitions and the number of distinct parts modulo 2 , respectively, then, $p_{1}^{2}(4)=p_{2}^{2}(4)=1$, and so $\tilde{p}(4)^{2}=6$. Therefore, the general formula of the number of overpartitions such that no parts is divisible by $s$, would be

$$
\tilde{p}(n)^{s}=\sum_{i} 2^{i} p_{i}^{s}(n) .
$$

Next, we propose a sum formula for the inverted graded parafermion of order $4, \prod_{k=1}^{\infty} \frac{\left(1+x^{2 k-1}\right)}{\left(1-x^{2 k}\right)}$. We learn from Corteel that this generating was considered by Mac Mahon and others, and the associated Ferrers diagram is called 2-modular diagram [36], [37]. The formula we propose is given by following expression,

$$
\prod_{k=1}^{\infty} \frac{\left(1+x^{2 k-1}\right)}{\left(1-x^{2 k}\right)}=1+\sum_{n=1, n \neq 2}^{\infty} \sum_{m \geq 1} \sum_{n_{1}, \cdots n_{m}} \frac{x^{n}(1+x)^{m}}{\left(1-x^{n_{1}}\right)\left(1-x^{n_{2}}\right) \cdots\left(1-x^{n_{m}}\right)},
$$

where $m$, is chosen such that $n+m$, is even and $n>m$ except when $n=1, m=1$. The exponents $n_{j}$, are even and distinct parts solutions to $n_{1}+n_{2}+\cdots+n_{m}=n+m$. Setting $s=4$, in equation (63), then the counter part of the above formula in the multiplicative number theory, would be

$$
\frac{\zeta(t) \zeta(4 t)}{\zeta(2 t)}=\sum_{n=1}^{\infty} \frac{1}{n^{t}}
$$

where the exponents in $n=\prod_{i} p_{i}^{r_{i}}, r_{i}$, are congruent to 0,1 modulo 4 .

Acknowledgments: I would like to thank S.Corteel for the combinatorial interpretation given to the formulas 80 and 81, K.S.Narain, A.Lascoux, M.O'Loughlin, G.Thompson, for critical reading of the manuscript and discussions, M.Somos, and D.Spector for correspondence and D.Zagier for his kind suggestion on formula (77), F.Diamond for correspondence on modular forms, and the Abdus Salam Centre For Theoretical Physics, Trieste Italy for support and hospitality throughout these years. The author is also grateful to the referee who examined the previous version thoroughly and offered numerous invaluable suggestions. 


\section{References}

[1] B. Julia, J. Phys. (France) 50,1371 (1989)

[2] D. Spector, comm. Math. Phys. 127,239 (1990).

[3] I. Bakas and M. Bowiwick, J. Math. Phys. 32,1881 (1991).

[4] D. Spector, J. Math. Phys. 32,1919 (1998).

[5] T. M. Apostol, An introduction to Analytic Number Theory (Springer-Verlag, New-York,1976).

[6] K.Kademova and M. Kraev, .Nuclear Physics,B26, 342 (1971).

[7] H.S. Green, (1953).Physical Review,90, 270 (1953)

[8] G. E . Andrews, The Theory Of Partitions,(Addison-Wesley,1976).

[9] J. A. Sellers Journal of Integer Sequences. 7 (2004) Article 04.2.4

[10] N. J. A.Sloane,The On-Line Encyclopedia of Integer Sequences.Published electronically at http://www.research.att.com/ njas/sequences/.

[11] S.Corteel and J.Lovejoy, Trans.Amer.Soc.356 1623 (2004)

[12] J.F.Fortin, P.Jacob and P.Mathieu, Ramanujan J. 10, 215 (2005).

[13] J-F Fortin et al J. Phys. A: Math. Gen. 381699 (2005)

[14] Michael P. Zalete and Roger S. Mong Phys. Rev. B 86, 245305 (2012)

[15] E. Witten, Nucl. Phys. B202253 (1982).

[16] Y. Ohnuki, M. Yamada, and S. Kamefuchi, Phys. Letters 36B, 51 (1971)

[17] V. L. Safonov,Phys. Stat. Sol. (b) 167 (1991), 109

[18] M. Hama, M. Sawamura, and H. Suzuki, Prog. Theor. Phys. 88, 149 (1992)

[19] B.Zwiebah, A First course in string theory, cambridge university press, (2005).

[20] I. Schur,Gesmmelte Abhandlungen. (Springer-Verlag,Berlin,1243 1973).

[21] I. Kani and C.Vafa Commun. Math.Phys. 130,529 (1990)

[22] I.J. Slater, Proc.London Math.Soc.54 147 (1952)

[23] A.V.Sills, The Electronic Journal of Combinatorics.10 1 (2003)

[24] G.H.Hardy and Wright An Intoduction The Theory Of numbers, 4th ed. (Oxford Clarendon Press, (1960)

[25] Neal Koblitz, Introduction to Elliptic Curves and Modular Forms, Springer-Verlag (1984)

[26] M. L. Glasser, J . Math. Phys. 14, 409 (1973)

[27] Jean-Piere Serre, Cours d'arithmetique, Press Universitaire de France, $3^{e}$ edition (1988) 
[28] S. Chaturvedi, V. Srinivasan, Phys. Lett. A 224, 249 (1997)

[29] I.G. Macdonald, Symmetric functions and Hall polynomials Clarendon, Oxford. (1995).

[30] O.W. Greenberg and A.M.L. Messiah, J. Math. Phys. 6500 (1965).

[31] M. Hama, M. Sawamura, and H. Suzuki, Prog. Theor. Phys. 861141 (1991).

[32] Y. Ohnuki and S. Kamefuchi, Quantum Field Theory and Parastatistics University of Tokyo Press/Springer Verlag, (1982)

[33] M. Hama, M. Sawamura, and H. Suzuki Europhys. Lett, 21383 (1993)

[34] P. Hagis, Trans. Amer. Math. Soc. 155, 375 (1971).

[35] Blencowe, Miles P.; Koshnick, Nicholas C.J. Math Phys, 425713 (2001)

[36] A.Berkovich and FG.Garvan, J. Combin. Theory Ser. A 100, 61 (2002)

[37] I.Pak, Ramanujan J. 12, 52006. 